\title{
ANALYSIS AND APPROXIMATION OF A STRAIN-LIMITING NONLINEAR ELASTIC MODEL
}

\author{
MIROSLAV BULÍČEK, JOSEF MÁLEK, AND ENDRE SÜLI
}

\begin{abstract}
Elastic solids with strain-limiting response to external loading represent an interesting class of material models, capable of describing stress concentration at strains with small magnitude. A theoretical justification of this class of models comes naturally from implicit constitutive theory. We investigate mathematical properties of static deformations for such strain-limiting nonlinear models. Focusing on the spatially periodic setting, we obtain results concerning existence, uniqueness and regularity of weak solutions, and existence of renormalized solutions for the full range of the positive scalar parameter featuring in the model. These solutions are constructed via a Fourier spectral method. We formulate a sufficient condition for ensuring that a renormalized solution is in fact a weak solution, and we comment on the extension of the analysis to non-periodic boundary-value problems.
\end{abstract}

\section{Dedicated to Professor K. R. Rajagopal}

\section{INTRODUCTION}

The recently developed implicit constitutive theory (see [11], [12]) expands quite considerably the possibilities for describing nonlinear responses of materials, even though the quantities involved in implicit constitutive models are the same as in classical linear models, which bear the names of Hooke, Lamé, Navier, Stokes, Darcy, and others. Another significant feature of the implicit constitutive theory is that it provides a firm theoretical foundation for various models in fluid and solid mechanics that were proposed by engineers, physicists and chemists in an ad hoc manner.

Concerning solids, that are the subject of this study, one of the main achievements of implicit constitutive theory is in providing a theoretical background and justification for nonlinear models involving the linearized strain; see [16]. In particular, it is thus possible to have models in which the linearized strain is in all circumstances a bounded function, even when the stress is very large. This class of implicit constitutive models, developed by Rajagopal in [14, 15], and which are referred to as limiting strain models, has the potential to be useful in describing the behavior of brittle materials near crack tips or notches, or concentrated loads inside the body or on its boundary. Both of these effects lead to stress concentration even though the gradient of the displacement is small. It is these limiting linearized strain models ${ }^{1}$ that are the subject of the present study, which is focused on the mathematical analysis of the existence and uniqueness of solutions to boundary-value problems in these models.

Limiting strain models have been thus far studied in several situations. Firstly, in the case of special deformations such as shearing, compressions, torsion, etc., Rajagopal himself, and Bustamante and Rajagopal aimed to assess whether the models exhibit the expected responses (cf. $[3],[15],[14]$ ). Secondly, in the case of anti-plane strain (stress) problems, considered in domains

Date: February 27, 2014.

The authors acknowledge the support of the ERC-CZ project LL1202 financed by MŠMT. E. Süli was also supported by the EPSRC Science and Innovation award to the Oxford Centre for Nonlinear PDE (EP/E035027/1).

${ }^{1}$ Models with limiting finite strain are found to be useful in modeling the response of various soft tissues that exhibit the phenomenon of finite extensibility. Also, Rajagopal's elastic models stemming from implicit constitutive theory seem to provide good description of Fung's experimental data concerning the passive response of biological tissues that indicate that the stress/strain response of the tissue is, to a good approximation, exponential. Referring to Fung's experimental results, Freed in his book [5] states: "Hooke's law is applicable for infinitesimal strains. Fung's law is applicable for moderate strains. The implicit theory of elasticity, or Rajagopal's elasticity, is applicable at finite strains." We refer also to [6] for another important application of elastic models based on implicit constitutive theory. 
with nonconvex cross-sections (including thus the domains with V-notches or cracks), the resulting scalar problem in two space dimensions has been analyzed by methods of asymptotic analysis in [19], by performing systematic computational tests in [9], and by analytical methods from the theory of nonlinear partial differential equations in [2]; the last result establishes the existence of weak solutions in nonconvex domains for values of the model parameter $r$ in the range $r \in(0,2)$, see equation (4) below, and in convex domains for the range $r \in(0, \infty)$. Thirdly, a detailed computational study of the complete problem in planar domains was performed in Ortiz et al. [10].

The present work is the first one with focus on the mathematical analysis of general boundaryvalue problems (which include systems of $\frac{1}{2} d(d+3)$ time-independent nonlinear partial differential equations of first order), featuring in limiting strain models, in bounded subsets of $\mathbb{R}^{d}, d \geq 2$.

\section{Formulation of the PRoblem, SIMPLifications AND Main RESUlts}

Let $\Omega \subset \mathbb{R}^{d}$ denote the set occupied by an elastic body being at a static state achieved as a result of the action of body forces $f: \Omega \rightarrow \mathbb{R}^{d}$ and traction forces $g: \Gamma_{N} \rightarrow \mathbb{R}^{d}$ whereas we assume that the boundary $\partial \Omega$ of the set $\Omega$ consists of two parts, $\Gamma_{N}$ and $\Gamma_{D}$, and the displacement $u: \Omega \rightarrow \mathbb{R}^{d}$ is given on $\Gamma_{D}$. Assuming that the response of the body is described by a constitutive equation relating the Cauchy stress tensor $T: \Omega \rightarrow \mathbb{R}_{\mathrm{sym}}^{d \times d}$ and the deformation gradient ${ }^{2}$ through the Galilean invariant Cauchy-Green deformation tensor $B$ implicitly (see [11], [12], [13]), then we arrive at the following problem: find $u$ and $T$ such that

$$
\begin{array}{rlrl}
-\operatorname{div} T=f, & G(T, B)=0 & & \text { in } \Omega, \\
T n=g & & \text { on } \Gamma_{N}, \\
u & =0 & & \text { on } \Gamma_{D},
\end{array}
$$

where $G: \mathbb{R}_{\mathrm{sym}}^{d \times d} \times \mathbb{R}_{\mathrm{sym}}^{d \times d} \rightarrow \mathbb{R}_{\mathrm{sym}}^{d \times d}$ is given and $n$ stands for the outer unit normal vector to the boundary of $\Omega$. One should consider only those relations $G(T, B)=0$ that are thermodynamically consistent, which means that the relations $G(T, B)=0$ should automatically guarantee that there is no dissipation of energy associated with the class of materials considered and the responses considered are elastic. This compatibility of the model with the laws of thermodynamics is addressed in [17] and [18].

Problem (1) includes as a special case models described by explicit relations of the form

$$
B=H(T) .
$$

If the material is isotropic, then a representation theorem leads to an expression of the form

$$
B=\alpha_{0} I+\alpha_{1} T+\alpha_{2} T^{2},
$$

where $\alpha_{i}, i=0,1,2$, are functions of the invariants of $T$, i.e., $\alpha_{i}=\alpha_{i}\left(\operatorname{tr} T, \operatorname{tr} T^{2}, \operatorname{tr} T^{3}\right)$.

If we assume that the displacement gradient is small in the sense that $\sup _{x \in \Omega}|\nabla u(x)| \ll 1$, and if we set $\alpha_{2}=0$, we obtain

$$
\varepsilon=\alpha_{0} I+\alpha_{1} T \quad \text { with } \quad \varepsilon=\varepsilon(u)=\frac{1}{2}\left(\nabla u+(\nabla u)^{T}\right) .
$$

Within this framework Rajagopal proposed (see [15], [14]) several limiting strain models that can be described by the relation

$$
\varepsilon=\alpha_{0}\left(\operatorname{tr} T, \operatorname{tr} T^{2}\right) I+\frac{T}{\mu_{0}\left(1+\left(\operatorname{tr} T^{2}\right)^{r / 2}\right)^{1 / r}}=\alpha_{0}\left(\operatorname{tr} T, \operatorname{tr} T^{2}\right) I+\frac{T}{\mu_{0}\left(1+|T|^{r}\right)^{1 / r}},
$$

which we aim to analyze. The parameter $\mu_{0}$ is a positive constant.

\footnotetext{
${ }^{2}$ Since we are studying a simplified problem throughout the whole paper, we restrict ourselves to introducing only those concepts that we need. Let $\mathcal{X} \mapsto \chi(\mathcal{X})=: x$ represent the motion of the body assigned to a typical point $\mathcal{X}$ in the reference configuration whose current position is $x$. We then define the displacement vector $u:=x-\mathcal{X}$, the deformation gradient $F:=\frac{\partial \chi}{\partial \mathcal{X}}$, the (left and right) Cauchy-Green deformation tensors $B=F F^{T}$ and $C=F^{T} F$, and the linearized strain tensor $\varepsilon=\varepsilon(u)=\frac{1}{2}\left(\nabla u+(\nabla u)^{T}\right)$.
} 
In this study, we make several simplifications, which we shall now specify. We shall also explain our reasons for doing so.

Firstly, we neglect the spherical part of the Cauchy stress, i.e. we set $\alpha_{0}=0$ in (2), and we deal only with one operator that encodes the key mathematical difficulties. Neglecting the spherical part of the Cauchy stress helps us to simplify the presentation. We believe that retaining the neglected spherical term will not alter the mathematical analysis in an essential way.

Secondly, we consider a domain of a special form: namely an axiparallel parallelepiped, with spatially periodic boundary conditions in the various co-ordinate directions. This essential simplification helps us to introduce not only the concept of weak solution to the problem under consideration, but also the concept of a renormalized solution. The spatially periodic setting also helps us to construct the solution via a specific numerical method, namely the Fourier spectral method. Thus our proof of existence of weak and renormalized solutions to the model is at the same time a proof of the convergence of the sequence of numerical approximations to the unknown analytical solution.

Thirdly, in the periodic setting the various bounds that are obtained on the sequence of approximating solutions are, in a sense, optimal, as there are no boundary effects, and the analysis therefore highlights the ideal objective that one should aim for in the case of other (mixed) boundary-value problems in general domains. We shall state some of the relevant open problems and conjectures concerning the extension of the present analysis to such nonperiodic boundaryvalue problems at the end of the paper.

These simplifications also allow us to provide a fairly complete picture regarding the existence and uniqueness of solutions for a nontrivial example of a strain-limiting nonlinear elastic model.

Since standard notations in continuum mechanics and in the theory of partial differential equations differ and since we are neglecting the spherical part of the Cauchy stress $T$, we make the following two changes to our notation: henceforth we shall write

$$
S \text { instead of } T \text { and } \quad D(u) \text { instead of } \varepsilon=\varepsilon(u) .
$$

We also set $\mu_{0}=1$.

The problem under consideration here is thus the following: suppose that $\Omega=(0,2 \pi)^{d}$, with $d \geq 2, r>0$ is a parameter in the model, and $f$ is a given $d$-component vector-function (the load-vector), which is $2 \pi$-periodic in each of the $d$ co-ordinate directions. The objective is to show the existence of a unique pair $(S, u)$, where $S$ is the stress tensor and $u$ is the displacement, which belong to suitable function spaces consisting of $d \times d$ matrix functions and $d$-component vector functions, respectively, that are $2 \pi$-periodic in each co-ordinate direction, such that

$$
-\operatorname{div} S=f
$$

and

$$
D(u)=S\left(1+|S|^{r}\right)^{-\frac{1}{r}} .
$$

In terms of the parameter $r$ featuring in the model the main results of the paper are the following:

(a) for $r \in\left(0, \frac{2}{d}\right)$, we prove the existence of a unique weak solution to the problem;

(b) for $r \in\left[\frac{2}{d}, \infty\right)$, we prove the existence of a renormalized solution to the problem, and we state sufficient conditions on the regularity of the stress tensor $S$, which imply that a renormalized solution is in fact a weak solution.

We note that despite its geometrical simplicity, one can still use the framework presented here to study the effects of concentrated loads that are active in the neighborhood of the center point of the periodic cell, assuming that the side-lengths of the cell are large enough so that the effects of concentrations are not effective in the neighbourhood of the boundary of the cell.

The paper is structured as follows. In Section 3 we define the sequence $\left\{\left(S_{N}, u_{N}\right)\right\}_{N \geq 1}$ of approximating solutions, which our subsequent weak compactness argument will be based upon, and we show the existence and uniqueness of the approximating solution for given $f$ and $r>0$. In Section 4 we prove appropriate bounds on $S_{N}$ and $u_{N}$, which then permit us to extract a 
subsequence from the sequence $\left\{\left(S_{N}, u_{N}\right)\right\}_{N \geq 1}$ that converges to a limiting object $(S, u)$, which is then identified as a weak solution to the problem under consideration. Having done so, we show that the weak solution is unique. The analysis in Section 4 relies on certain uniform bounds (with respect to $N)$ on $S_{N}$ and $u_{N}$, which are only valid for $r \in\left(0, \frac{2}{d}\right)$. In order to show the existence of solutions for $r \in\left[\frac{2}{d}, \infty\right)$, we shall introduce in Section 5 the notion of renormalized solution to the problem and will prove the existence of a renormalized solution for all $r \in\left[\frac{2}{d}, \infty\right)$. As was already noted above, we shall also state conditions on the regularity of the stress tensor $S$ that suffice to ensure that a renormalized solution is in fact a weak solution. In the Conclusion, we relate the results achieved in the spatially periodic setting to possible results and open problems regarding the analysis of the more general problem (1). The paper closes with an Appendix, which contains the proofs of various Korn-type inequalities in periodic Lebesgue spaces, that we were unable to find in the literature and have therefore decided to include for the sake of completeness.

\section{Definition of the Approximation: existence AND Uniqueness of solutions}

Consider the domain $\Omega:=(0,2 \pi)^{d}$ in $\mathbb{R}^{d}, d \geq 2$. All function spaces consisting of real-valued $2 \pi$-periodic functions (by which we mean $2 \pi$-periodic in each of the $d$ co-ordinate directions) will be labelled with the subscript \#; subspaces of these, consisting of $2 \pi$-periodic functions whose integral over $\Omega$ is equal to 0 , will be labelled with the subscript $*$; in order to avoid notational clutter we shall not use the symbols \# and $*$ in the various norm signs. It will be clear from the argument of the norm which of the symbols \# or $*$ is intended. For example, $L_{\#}^{p}(\Omega)$ will denote the Lebesgue space of all real-valued $2 \pi$-periodic functions $v$ such that $|v|^{p}$ is integrable of $\Omega$, equipped with the norm $\|\cdot\|_{L^{p}(\Omega)}$. It is understood that the usual modification is made when $p=\infty$. Spaces of $d$-component vector functions, where each component belongs to a certain function space $X$, will be denoted by $[X]^{d}$, while spaces of $d \times d$ component matrix functions each of whose components is an element of $X$ will be signified by $[X]^{d \times d}$. Letting $C_{\#}^{\infty}(\bar{\Omega})$ denote the linear space consisting of the restriction to $\bar{\Omega}$ of all real-valued $2 \pi$-periodic $C^{\infty}$ functions defined on $\mathbb{R}^{d}$, we note that $C_{\#}^{\infty}(\bar{\Omega})$ is dense in $L_{\#}^{p}(\Omega)$ for all $p \in[1, \infty)$; analogously, $C_{*}^{\infty}(\bar{\Omega})$ is dense in $L_{*}^{p}(\Omega)$ for $1 \leq p<\infty$. The Sobolev space $W_{\#}^{1, p}(\Omega), 1 \leq p<\infty$, will be defined as the closure of $C_{\#}^{\infty}(\bar{\Omega})$ in the Sobolev norm $\|\cdot\|_{W^{1, p}(\Omega)}$, where

$$
\|v\|_{W^{1, p}(\Omega)}:=\left(\|v\|_{L^{p}(\Omega)}^{p}+\|\nabla v\|_{L^{p}(\Omega)}^{p}\right)^{\frac{1}{p}}
$$

here, $\|\nabla v\|_{L^{p}(\Omega)}:=\||\nabla v|\|_{L^{p}(\Omega)}$, where $|\nabla v|$ denotes the Euclidean norm of $\nabla v$. Analogously, $W_{*}^{1, p}(\Omega), 1 \leq p<\infty$, will be defined as the closure of $C_{*}^{\infty}(\bar{\Omega})$ in the Sobolev norm $\|\cdot\|_{W^{1, p}(\Omega)}$. In the case of a $d$-component vector-valued function $v$, the definition of the norm $\|v\|_{W^{1, p}(\Omega)}$ is the same as above, except that $\|v\|_{L^{p}(\Omega)}:=\||v|\|_{L^{p}(\Omega)}$, with $|\cdot|$ again signifying the Euclidean norm, while $\|\nabla v\|_{L^{p}(\Omega)}:=\||\nabla v|\|_{L^{p}(\Omega)}$, where now $|\nabla v|$ denotes the Frobenius norm of the $d \times d$ matrix $\nabla v$. We recall that the Frobenius norm on $\mathbb{R}^{d \times d}$ is defined by $|X|^{2}:=X: X=\operatorname{tr}\left(X^{\mathrm{T}} X\right)$.

We further define

$$
H_{\#}(\operatorname{div} ; \Omega):=\left\{v \in\left[L_{\#}^{2}(\Omega)\right]^{d}: \text { such that } \operatorname{div} v \in L_{\#}^{2}(\Omega)\right\},
$$

equipped with the norm

$$
\|v\|_{H(\operatorname{div} ; \Omega)}:=\left(\|v\|_{L^{2}(\Omega)}^{2}+\|\operatorname{div} v\|_{L^{2}(\Omega)}^{2}\right)^{\frac{1}{2}}
$$

Let

$$
\Sigma_{N} \subset H_{\#, \operatorname{symm}}(\operatorname{div} ; \Omega):=\left\{S \in\left[L_{\#}^{2}(\Omega)\right]^{d \times d}: S=S^{\mathrm{T}}, \operatorname{div} S \in\left[L_{\#}^{2}(\Omega)\right]^{d}\right\},
$$

equipped with norm

and

$$
\|S\|_{H(\operatorname{div} ; \Omega)}:=\left(\|S\|_{L^{2}(\Omega)}^{2}+\|\operatorname{div} S\|_{L^{2}(\Omega)}^{2}\right)^{\frac{1}{2}}
$$

$$
V_{N} \subset\left[W_{*}^{1,2}(\Omega)\right]^{d}=\left\{v \in\left[W_{\#}^{1,2}(\Omega)\right]^{d}: \int_{\Omega} v(x) \mathrm{d} x=0\right\}
$$


be a pair of finite-dimensional spaces consisting of, respectively, $\mathbb{R}^{d \times d}$-valued and $\mathbb{R}^{d}$-valued functions, whose components are $2 \pi$-periodic real-valued trigonometric polynomials of degree $N$, $N \geq 1$, in each of the $d$-coordinate directions. The pair of spaces $\left(\Sigma_{N}, V_{N}\right)$ satisfies the following inf-sup condition: let $b(v, T):=-(v, \operatorname{div} T)$; then, there exists a positive constant $c_{\text {inf-sup }}$, independent of $N$, such that

$$
\inf _{v_{N} \in V_{N} \backslash\{0\}} \sup _{T_{N} \in \Sigma_{N} \backslash\{0\}} \frac{b\left(v_{N}, T_{N}\right)}{\left\|v_{N}\right\|_{L^{2}(\Omega)}\left\|T_{N}\right\|_{H(\operatorname{div} ; \Omega)}} \geq c_{\text {inf-sup }} .
$$

For a short proof of (5) we refer to the Appendix at the end of the paper, where it is also shown that $c_{\text {inf-sup }} \geq 1 / 3$.

Assume further that $r>0$ and define

$$
F(X):=X\left(1+|X|^{r}\right)^{-1 / r}, \quad X \in \mathbb{R}^{d \times d},
$$

where, again, $|\cdot|$ denotes the Frobenius norm on $\mathbb{R}^{d \times d}$.

Suppose that $f \in\left[W_{*}^{1, t}(\Omega)\right]^{d}=\left\{g \in\left[W_{\#}^{1, t}(\Omega)\right]^{d}: \int_{\Omega} g(x) \mathrm{d} x=0\right\}$, for some $t>1$. We consider the following discrete problem: find $\left(S_{N}, u_{N}\right) \in \Sigma_{N} \times V_{N}$ such that

$$
\begin{array}{rlrl}
-\left(\operatorname{div} S_{N}, v_{N}\right) & =\left(f, v_{N}\right) & & \forall v_{N} \in V_{N}, \\
\hat{D}_{N} & :=F\left(S_{N}\right), & & \\
\left(D\left(u_{N}\right), T_{N}\right) & =\left(\hat{D}_{N}, T_{N}\right) & \forall T_{N} \in \Sigma_{N} .
\end{array}
$$

Lemma 1. For any $y \geq 0$ and $r>0$, we have that

$$
\min \left(1,2^{-1+1 / r}\right)(1+y) \leq\left(1+y^{r}\right)^{1 / r} \leq \max \left(1,2^{-1+1 / r}\right)(1+y) .
$$

Proof. Consider the function $y \in[0, \infty) \mapsto g(y):=\left(1+y^{r}\right)^{1 / r} /(1+y) \in(0, \infty)$. Note that $g \in C([0, \infty)) \cap C^{\infty}((0, \infty)), g(0)=1, \lim _{y \rightarrow \infty} g(y)=1$, and

$$
g^{\prime}(y)=\frac{\left(1+y^{r}\right)^{-1+1 / r}}{(1+y)^{2}}\left(y^{r-1}-1\right) .
$$

Thus $g$ has a unique stationary point in the interval $(0, \infty)$, at $y=1$. As

$$
g(1)=2^{-1+1 / r} \begin{cases}\geq 1 & \text { if } 0<r \leq 1 \\ \leq 1 & \text { if } r \geq 1\end{cases}
$$

we deduce that $\max _{y \in[0, \infty)} g(y)=g(1)$ if $0<r \leq 1$ and $\max _{y \in[0, \infty)} g(y)=g(0)$ if $r \geq 1$. Hence the desired upper bound. Similarly, $\min _{y \in[0, \infty)} g(y)=\min \{g(0), g(1)\}$.

Lemma 2. Let $r>0$, and consider the mapping

$$
X \in \mathbb{R}^{d \times d} \mapsto F(X):=X\left(1+|X|^{r}\right)^{-1 / r} \in \mathbb{R}^{d \times d} .
$$

Then, for each $A, B \in \mathbb{R}^{d \times d}$, we have that

$$
|F(A)-F(B)| \leq 2|A-B|,
$$

and

$$
(F(A)-F(B)):(A-B) \geq \min \left(1,2^{r-1 / r}\right)|A-B|^{2}(1+|A|+|B|)^{-r-1} .
$$

Proof. We begin by observing that

$$
\begin{aligned}
F(A)-F(B) & =\int_{0}^{1} \frac{\mathrm{d}}{\mathrm{d} \theta} F(\theta A+(1-\theta) B) \mathrm{d} \theta \\
& =\int_{0}^{1} \frac{\mathrm{d}}{\mathrm{d} \theta}\left[(\theta A+(1-\theta) B)\left(1+|\theta A+(1-\theta) B|^{r}\right)^{-1 / r}\right] \mathrm{d} \theta
\end{aligned}
$$


Thanks to the definition of the matrix norm $|\cdot|$, we have that

$$
\begin{aligned}
& \frac{\mathrm{d}}{\mathrm{d} \theta}\left[(\theta A+(1-\theta) B)\left(1+|\theta A+(1-\theta) B|^{r}\right)^{-1 / r}\right]=\frac{A-B}{\left(1+|\theta A+(1-\theta) B|^{r}\right)^{1 / r}} \\
& -(\theta A+(1-\theta) B) \frac{|\theta A+(1-\theta) B|^{r-1}}{\left(1+|\theta A+(1-\theta) B|^{r}\right)^{1+1 / r}} \frac{(\theta A+(1-\theta) B):(A-B)}{|\theta A+(1-\theta) B|} .
\end{aligned}
$$

Hence, for any $A, B, C \in \mathbb{R}^{d \times d}$, we have that

$$
\begin{aligned}
& (F(A)-F(B)): C=\int_{0}^{1} \frac{(A-B): C}{\left(1+|\theta A+(1-\theta) B|^{r}\right)^{1 / r}} \mathrm{~d} \theta \\
& \quad-\int_{0}^{1}[(\theta A+(1-\theta) B): C][(\theta A+(1-\theta) B):(A-B)] \frac{|\theta A+(1-\theta) B|^{r-2}}{\left(1+|\theta A+(1-\theta) B|^{r}\right)^{1+1 / r}} \mathrm{~d} \theta,
\end{aligned}
$$

and therefore, by applying the Cauchy-Schwarz inequality on the right-hand side,

$$
|F(A)-F(B)| \leq 2|A-B| \quad \forall A, B \in \mathbb{R}^{d \times d} .
$$

The proof of the second inequality in the statement of the lemma proceeds similarly:

$$
\begin{aligned}
(F(A)-F(B)):(A-B)= & \int_{0}^{1} \frac{|A-B|^{2}}{\left(1+|\theta A+(1-\theta) B|^{r}\right)^{1 / r}} \mathrm{~d} \theta \\
& -\int_{0}^{1}[(\theta A+(1-\theta) B):(A-B)]^{2} \frac{|\theta A+(1-\theta) B|^{r-2}}{\left(1+|\theta A+(1-\theta) B|^{r}\right)^{1+1 / r}} \mathrm{~d} \theta,
\end{aligned}
$$

and by the Cauchy-Schwarz inequality

$$
[(A-B):(\theta A+(1-\theta) B)]^{2} \leq|A-B|^{2}|\theta A+(1-\theta) B|^{2} .
$$

Thus,

$$
\begin{aligned}
(F(A)-F(B)):(A-B) \geq & \int_{0}^{1} \frac{|A-B|^{2}}{\left(1+|\theta A+(1-\theta) B|^{r}\right)^{1 / r}} \mathrm{~d} \theta \\
& \left.-\int_{0}^{1}|A-B|^{2} \mid \theta A+(1-\theta) B\right)\left.\right|^{2} \frac{|\theta A+(1-\theta) B|^{r-2}}{\left(1+|\theta A+(1-\theta) B|^{r}\right)^{1+1 / r}} \mathrm{~d} \theta \\
= & |A-B|^{2} \int_{0}^{1}\left(1+|\theta A+(1-\theta) B|^{r}\right)^{-1-1 / r} \mathrm{~d} \theta .
\end{aligned}
$$

It follows from Lemma 1 that, for any $\theta \in[0,1]$ and any $A, B \in \mathbb{R}^{d \times d}$,

$$
\begin{aligned}
\left(1+|\theta A+(1-\theta) B|^{r}\right)^{1 / r} & \leq \max \left(1,2^{-1+1 / r}\right)(1+|\theta A+(1-\theta) B|) \\
& \leq \max \left(1,2^{-1+1 / r}\right)(1+|A|+|B|) .
\end{aligned}
$$

Hence

$$
\left(1+|\theta A+(1-\theta) B|^{r}\right)^{(r+1) / r} \leq \max \left(1,2^{1 / r-r}\right)(1+|A|+|B|)^{r+1} .
$$

Applying this to the integrand, we deduce that

$$
(F(A)-F(B)):(A-B) \geq \min \left(1,2^{r-1 / r}\right)|A-B|^{2}(1+|A|+|B|)^{-r-1},
$$

which is the second inequality in the statement of the lemma.

With these preliminary results in place, we are now ready to embark on the proof of existence and uniqueness of solutions to the discrete problem (6)-(8).

\section{Existence and uniqueness of solutions}

Assuming for the moment the existence of a solution $\left(S_{N}, u_{N}\right) \in \Sigma_{N} \times V_{N}$ to (6)-(8), we shall show that the solution must be unique. Suppose otherwise, that there exist $\left(S_{N}^{i}, u_{N}^{i}\right) \in \Sigma_{N} \times V_{N}$ that solve (6)-(8) for $i=1,2$. Hence,

$$
-\left(\operatorname{div}\left(S_{N}^{1}-S_{N}^{2}\right), v_{N}\right)-\left(D\left(u_{N}^{1}-u_{N}^{2}\right), T_{N}\right)+\left(F\left(S_{N}^{1}\right)-F\left(S_{N}^{2}\right), T_{N}\right)=0
$$


for all $\left(T_{N}, v_{N}\right) \in \Sigma_{N} \times V_{N}$. We take $T_{N}=S_{N}^{1}-S_{N}^{2}$ and $v_{N}=u_{N}^{1}-u_{N}^{2}$, and note that, after partial integration in the first term,

$$
\begin{aligned}
-(\operatorname{div} & \left.\left(S_{N}^{1}-S_{N}^{2}\right), u_{N}^{1}-u_{N}^{2}\right)-\left(D\left(u_{N}^{1}-u_{N}^{2}\right), S_{N}^{1}-S_{N}^{2}\right) \\
\quad= & \left(S_{N}^{1}-S_{N}^{2}, \nabla\left(u_{N}^{1}-u_{N}^{2}\right)\right)-\left(D\left(u_{N}^{1}-u_{N}^{2}\right), S_{N}^{1}-S_{N}^{2}\right) \\
\quad= & \left(S_{N}^{1}-S_{N}^{2}, D\left(u_{N}^{1}-u_{N}^{2}\right)\right)-\left(D\left(u_{N}^{1}-u_{N}^{2}\right), S_{N}^{1}-S_{N}^{2}\right)=0 .
\end{aligned}
$$

Consequently,

$$
\left(F\left(S_{N}^{1}\right)-F\left(S_{N}^{2}\right), S_{N}^{1}-S_{N}^{2}\right)=0 .
$$

Lemma 2 then implies that $S_{N}^{1} \equiv S_{N}^{2}$ on $\Omega$, and hence $\hat{D}_{N}^{1} \equiv \hat{D}_{N}^{2}$ on $\Omega$, which yields that $D\left(u_{N}^{1}-u_{N}^{2}\right) \equiv 0$ on $\Omega$. By Korn's inequality (cf. Lemma A.2), we then have that $u_{N}^{1}-u_{N}^{2} \equiv 0$ on $\Omega$, thus completing the proof of uniqueness of the solution to discrete problem $(6)-(8)$.

Next we prove the existence of a solution $\left(S_{N}, u_{N}\right)$ to (6)-(8). First we choose any $\hat{S}_{N} \in \Sigma_{N}$ such that $-\left(\operatorname{div} \hat{S}_{N}, v_{N}\right)=\left(f, v_{N}\right)$ for all $v_{N} \in V_{N}$, and let $S_{N, 0}:=S_{N}-\hat{S}_{N}$. The existence of such an $\hat{S}_{N}$ will be shown below; for the time being, we shall proceed by taking the existence of such an $\hat{S}_{N}$ for granted. Clearly, $-\left(\operatorname{div} S_{N, 0}, v_{N}\right)=0$ for all $v_{N} \in V_{N}$, which then motivates us to define

$$
\Sigma_{N, 0}:=\left\{T_{N} \in \Sigma_{N}:-\left(\operatorname{div} T_{N}, v_{N}\right)=0 \text { for all } v_{N} \in V_{N}\right\}
$$

As $0 \in \Sigma_{N, 0}$, the set $\Sigma_{N, 0}$ is nonempty. Problem (6)-(8) can be therefore restated in the following equivalent form: find $\left(S_{N, 0}, u_{N}\right) \in \Sigma_{N, 0} \times V_{N}$ such that

$$
\left(D\left(u_{N}\right), T_{N}\right)=\left(F\left(S_{N, 0}+\hat{S}_{N}\right), T_{N}\right) \quad \forall T_{N} \in \Sigma_{N}
$$

Now, for $T_{N} \in \Sigma_{N, 0},\left(D\left(v_{N}\right), T_{N}\right)=\left(\nabla v_{N}, T_{N}\right)=-\left(v_{N}, \operatorname{div} T_{N}\right)=-\left(\operatorname{div} T_{N}, v_{N}\right)=0$ for all $v_{N} \in V_{N}$. Hence, (9) indicates that we should seek $S_{N, 0} \in \Sigma_{N, 0}$ such that

$$
\left(F\left(S_{N, 0}+\hat{S}_{N}\right), T_{N}\right)=0 \quad \forall T_{N} \in \Sigma_{N, 0}
$$

Let us consider the nonlinear operator $\mathfrak{F}: \Sigma_{N, 0} \rightarrow \Sigma_{N, 0}$, defined on the finite-dimensional Hilbert space $\Sigma_{N, 0}$, equipped with the inner product and norm of $\left[L_{\#}^{2}(\Omega)\right]^{d \times d}$, by

$$
\mathfrak{F}\left(U_{N}\right):=P_{N} F\left(U_{N}+\hat{S}_{N}\right), \quad U_{N} \in \Sigma_{N, 0},
$$

where $P_{N}$ denotes the orthogonal projector in $\left[L_{\#}^{2}(\Omega)\right]^{d \times d}$ onto $\Sigma_{N, 0}$.

Thanks to the first inequality in the statement of Lemma 2, we then have that

$$
\left\|\mathfrak{F}\left(U_{N}^{1}\right)-\mathfrak{F}\left(U_{N}^{2}\right)\right\|_{L^{2}(\Omega)} \leq 2\left\|U_{N}^{1}-U_{N}^{2}\right\|_{L^{2}(\Omega)} \quad \forall U_{N}^{1}, U_{N}^{2} \in \Sigma_{N, 0},
$$

and therefore $\mathfrak{F}: \Sigma_{N, 0} \rightarrow \Sigma_{N, 0}$ is (globally) Lipschitz continuous on $\Sigma_{N, 0}$.

Note further that

$$
\left(\mathfrak{F}\left(U_{N}\right), U_{N}\right)=\left(\frac{U_{N}+\hat{S}_{N}}{\left(1+\left|U_{N}+\hat{S}_{N}\right|^{r}\right)^{1 / r}}, U_{N}\right) \geq \frac{1}{2} \int_{\Omega} \frac{\left|U_{N}\right|^{2}-\left|\hat{S}_{N}\right|^{2}}{\left(1+\left|U_{N}+\hat{S}_{N}\right|^{r}\right)^{1 / r}} \mathrm{~d} x .
$$

Thus, thanks to Lemma 1 and noting that $\left(1+\left|U_{N}+\hat{S}_{N}\right|^{r}\right)^{1 / r} \geq 1$, we have that

$$
\left(\mathfrak{F}\left(U_{N}\right), U_{N}\right) \geq \frac{1}{2} \min \left(1,2^{1-1 / r}\right) \int_{\Omega} \frac{\left|U_{N}\right|^{2}}{1+\left|U_{N}\right|+\left|\hat{S}_{N}\right|} \mathrm{d} x-\frac{1}{2} \int_{\Omega}\left|\hat{S}_{N}\right|^{2} \mathrm{~d} x .
$$

Now,

$$
\frac{\left|U_{N}\right|^{2}}{1+\left|U_{N}\right|+\left|\hat{S}_{N}\right|} \geq \frac{\left|U_{N}\right|^{2}}{\left(1+\left\|\hat{S}_{N}\right\|_{L^{\infty}(\Omega)}\right)\left(1+\left\|U_{N}\right\|_{L^{\infty}(\Omega)}\right)},
$$

and therefore, by the Nikol'skiu inequality $\left\|U_{N}\right\|_{L^{\infty}(\Omega)} \leq C_{\mathrm{inv}} N^{d / 2}\left\|U_{N}\right\|_{L^{2}(\Omega)}$, we deduce that

$$
\left(\mathfrak{F}\left(U_{N}\right), U_{N}\right) \geq \frac{1}{2} \frac{\min \left(1,2^{1-1 / r}\right)}{1+\left\|\hat{S}_{N}\right\|_{L^{\infty}(\Omega)}} \frac{1}{1+C_{\mathrm{inv}} N^{d / 2}\left\|U_{N}\right\|_{L^{2}(\Omega)}}\left\|U_{N}\right\|_{L^{2}(\Omega)}^{2}-\frac{1}{2}\left\|\hat{S}_{N}\right\|_{L^{2}(\Omega)}^{2} .
$$


Thus, for any $U_{N} \in \Sigma_{N, 0}$ such that $\left\|U_{N}\right\|_{L^{2}(\Omega)}=\mu>0$, we have that

$$
\left(\mathfrak{F}\left(U_{N}\right), U_{N}\right) \geq \frac{1}{2} \frac{\min \left(1,2^{1-1 / r}\right)}{1+\left\|\hat{S}_{N}\right\|_{L^{\infty}(\Omega)}} \frac{\mu^{2}}{1+C_{\mathrm{inv}} N^{d / 2} \mu}-\frac{1}{2}\left\|\hat{S}_{N}\right\|_{L^{2}(\Omega)}^{2} .
$$

For $N \geq 1$ fixed (and therefore $\left\|\hat{S}_{N}\right\|_{L^{\infty}(\Omega)}$ and $\left\|\hat{S}_{N}\right\|_{L^{2}(\Omega)}$ also fixed), the expression on the right-hand side of the last displayed inequality is a continuous function of $\mu \in(0, \infty)$, which converges to $+\infty$ as $\mu \rightarrow+\infty$; thus, there exists a $\mu_{0}=\mu_{0}\left(d, r, N,\left\|\hat{S}_{N}\right\|_{L^{\infty}(\Omega)},\left\|\hat{S}_{N}\right\|_{L^{2}(\Omega)}\right)$, such that $\left(\mathfrak{F}\left(U_{N}\right), U_{N}\right)>0$ for all $U_{N} \in \Sigma_{N, 0}$ satisfying $\left\|U_{N}\right\|_{L^{2}(\Omega)}=\mu$, for $\mu>\mu_{0}$.

We shall invoke the following corollary of Brouwer's fixed point theorem (cf. Girault \& Raviart [8], Corollary 1.1, p.279).

Lemma 3. Let $\mathcal{H}$ be a finite-dimensional Hilbert space whose inner product is denoted by $(\cdot, \cdot)_{\mathcal{H}}$ and the corresponding norm by $\|\cdot\|_{\mathcal{H}}$. Let $\mathfrak{F}$ be a continuous mapping from $\mathcal{H}$ into $\mathcal{H}$ with the following property: there exists a $\mu>0$ such that $(\mathfrak{F}(v), v)_{\mathcal{H}}>0$ for all $v \in \mathcal{H}$ with $\|v\|_{\mathcal{H}}=\mu$. Then, there exists an element $u \in \mathcal{H}$ such that $\|u\|_{\mathcal{H}} \leq \mu$ and $\mathfrak{F}(u)=0$.

By taking $\mathcal{H}=\Sigma_{N, 0}$, equipped with the inner product and norm of $\left[L_{\#}^{2}(\Omega)\right]^{d \times d}$, we deduce from Lemma 3 the existence of an $S_{N, 0} \in \Sigma_{N, 0}$ that solves (10), and thus, recalling that $S_{N}=S_{N, 0}+\hat{S}_{N}$, we have also shown the existence of an $S_{N} \in \Sigma_{N} \operatorname{such}$ that $-\left(\operatorname{div} S_{N}, v_{N}\right)=\left(f, v_{N}\right)$ for all $v_{N} \in V_{N}$.

Having shown the existence of $S_{N} \in \Sigma_{N}$, we return to (9) in order to show the existence of a $u_{N} \in V_{N}$ such that

$$
\left(D\left(u_{N}\right), T_{N}\right)=\left(F\left(S_{N}\right), T_{N}\right) \quad \forall T_{N} \in \Sigma_{N} .
$$

Equivalently, we wish to show the existence of a $u_{N} \in V_{N}$ such that

$$
b\left(u_{N}, T_{N}\right)=\ell\left(T_{N}\right) \quad \forall T_{N} \in \Sigma_{N},
$$

where

$$
b\left(v_{N}, T_{N}\right):=-\left(v_{N}, \operatorname{div} T_{N}\right) \text { and } \quad \ell\left(T_{N}\right):=\left(F\left(S_{N}\right), T_{N}\right) .
$$

We note that $\ell\left(T_{N}\right)=0$ for all $T_{N} \in \Sigma_{N, 0}$, i.e., $\ell \in\left(\Sigma_{N, 0}\right)^{0}$ (the annihilator of $\left.\Sigma_{N, 0}\right)$. The existence of a unique $u_{N} \in V_{N}$ satisfying (11) then follows, thanks to the inf-sup condition (5), from the fundamental theorem of the theory of mixed variational problems stated in Lemma 4.1(ii) on p.40 of Girault \& Raviart [7].

At the very beginning of our proof of existence of solutions we postulated the existence of an $\hat{S}_{N} \in \Sigma_{N}$ such that $-\left(\operatorname{div} \hat{S}_{N}, v_{N}\right)=\left(f, v_{N}\right)$ for all $v_{N} \in V_{N}$. Part (iii) of Lemma 4.1 on p.40 of Girault \& Raviart [7] implies, again thanks to the inf-sup condition (5), the existence of an $\hat{S}_{N} \in \Sigma_{N}$ such that $b\left(v_{N}, \hat{S}_{N}\right)=\left(f, v_{N}\right)$ for all $v_{N} \in V_{N}$; i.e., $-\left(\operatorname{div} \hat{S}_{N}, v_{N}\right)=\left(f, v_{N}\right)$ for all $v_{N} \in V_{N}$. Thus we have proved both the existence and the uniqueness of solutions to the discrete problem (6)-(8).

\section{Convergence of the Sequence of approximate solutions}

It remains to show that the sequence of approximate solutions generated by (6)-(8) converges in a suitable sense, and that the limiting function is a (weak) solution to the problem under consideration. We define the function space

$$
D_{*}^{1, \infty}(\Omega):=\left\{w \in\left[L_{\#}^{1}(\Omega)\right]^{d}: D(w) \in\left[L_{\#}^{\infty}(\Omega)\right]^{d \times d}, \int_{\Omega} w(x) \mathrm{d} x=0\right\} .
$$

Trivially, $V_{N} \subset D_{*}^{1, \infty}(\Omega)$ for each $N \geq 1$. As, by Hölder's inequality, $\|D(w)\|_{L^{p}(\Omega)}<\infty$ for any $w \in D_{*}^{1, \infty}(\Omega)$ and any $p \in[1, \infty)$, Korn's inequality (cf. Lemma A.1) implies that the seminorm $w \in D_{*}^{1, \infty}(\Omega) \mapsto\|D(w)\|_{L^{\infty}(\Omega)}$ is in fact a norm on $D_{*}^{1, \infty}(\Omega)$.

Lemma 4. $\left[C_{*}^{\infty}(\bar{\Omega})\right]^{d}$ is weak-* dense in $D_{*}^{1, \infty}(\Omega)$ against $\left[L_{\#}^{1}(\Omega)\right]^{d \times d}$, in the sense that for each $v \in D_{*}^{1, \infty}(\Omega)$ there exists a sequence $\left\{v_{n}\right\}_{n \geq 1} \subset\left[C_{*}^{\infty}(\bar{\Omega})\right]^{d}$ such that

$$
\int_{\Omega} T(x): D\left(v_{n}(x)\right) \mathrm{d} x \stackrel{n \rightarrow \infty}{\rightarrow} \int_{\Omega} T(x): D(v(x)) \mathrm{d} x \quad \forall T \in\left[L_{\#}^{1}(\Omega)\right]^{d \times d} .
$$


Proof. Let $v \in\left[L_{*}^{1}(\Omega)\right]^{d}$. The function $v$ then has the Fourier series expansion

$$
v(x)=\sum_{k \in \mathbb{Z}^{d} \backslash\{0\}} \hat{v}(k) \mathrm{e}^{i k \cdot x}, \quad \text { where } \hat{v}(k):=\frac{1}{(2 \pi)^{d}} \int_{\Omega} v(x) \mathrm{e}^{-i k \cdot x} \mathrm{~d} x .
$$

Let $r:=\left(r_{1}, \ldots, r_{d}\right)$ with $0<r_{j}<1$ for $j=1, \ldots, d$, and, for a multi-index $m \in \mathbb{Z}^{d}$, define $r^{|m|}:=r_{1}^{m_{1}} \cdots r_{d}^{m_{d}}$. We consider the Poisson kernel:

$$
P_{r}(x):=\sum_{m \in \mathbb{Z}^{d}} r^{|m|} \mathrm{e}^{\imath m \cdot x}=\prod_{j=1}^{d} \frac{1-r_{j}^{2}}{1-2 r_{j} \cos x_{j}+r_{j}^{2}}, \quad x \in \Omega,
$$

and, given any $v \in D_{*}^{1, \infty}(\Omega)$, we define the Poisson integral of $v$ as $P_{r} * v$. We then take

$$
v_{n}(x):=P_{r_{n}} * v, \quad \text { with } r_{n}:=\left(1-\frac{1}{n}, \ldots, 1-\frac{1}{n}\right) \text { and } x \in \Omega,
$$

(so that $r_{n} \rightarrow(1, \ldots, 1)$ as $\left.n \rightarrow \infty\right)$, with the convolution $*$ understood in the sense that each of the $d$ components of $v$ is individually convolved with $P_{r_{n}}$. For each fixed $n \geq 1, v_{n} \in\left[C_{\#}^{\infty}(\bar{\Omega})\right]^{d}$. By the convolution theorem $\hat{v}_{n}(k)=(2 \pi)^{d} \widehat{P_{r_{n}}}(k) \hat{v}(k)$ for all $k \in \mathbb{Z}^{d}$, and therefore, as $0=$ $\int_{\Omega} v(x) \mathrm{d} x=(2 \pi)^{d} \hat{v}(0)$, also $\hat{v}_{n}(0)=0$, meaning that $\int_{\Omega} v_{n}(x) \mathrm{d} x=0$; i.e., $\left\{v_{n}\right\}_{n \geq 1} \subset\left[C_{*}^{\infty}(\bar{\Omega})\right]^{d}$. Furthermore, by properties of the convolution, $D\left(v_{n}\right)=D\left(P_{r_{n}} * v\right)=P_{r_{n}} * D(v)$. Note also that $P_{r_{n}}(x)=P_{r_{n}}(-x)$ for all $x \in \Omega$ and each $n=1,2 \ldots$ Thus we have that

$$
\begin{aligned}
& \left|\int_{\Omega} T(x):\left[P_{r_{n}}(x) * D(v(x))\right] \mathrm{d} x-\int_{\Omega} T(x): D(v(x)) \mathrm{d} x\right| \\
& \quad=\left|\int_{\Omega}\left[P_{r_{n}}(x) * T(x)-T(x)\right]: D(v(x)) \mathrm{d} x\right| \leq\left\|P_{r_{n}} * T-T\right\|_{L^{1}(\Omega)}\|D(v)\|_{L^{\infty}(\Omega)} .
\end{aligned}
$$

By Corollary 2.15 to Theorem 2.11 on p.256 and p.253, respectively, of Stein \& Weiss [21], we have that $\left\|P_{r_{n}} * T-T\right\|_{L^{1}(\Omega)} \rightarrow 0$ as $n \rightarrow \infty$. Hence,

$$
\begin{gathered}
\int_{\Omega} T(x): D\left(v_{n}(x)\right) \mathrm{d} x=\int_{\Omega} T(x):\left[P_{r_{n}}(x) * D(v(x))\right] \mathrm{d} x \\
\stackrel{n \rightarrow \infty}{\rightarrow} \int_{\Omega} T(x): D(v(x)) \mathrm{d} x \quad \forall T \in\left[L_{\#}^{1}(\Omega)\right]^{d \times d} .
\end{gathered}
$$

That completes the proof.

Theorem 1. Suppose that $f \in\left[{W_{*}^{1, t}}^{1,}(\Omega)\right]^{d}$ for some $t>1$; then, there exists a unique pair $(S, u) \in\left[L_{\#}^{1}(\Omega)\right]^{d \times d} \times D_{*}^{1, \infty}(\Omega)$, such that

$$
(S, D(v))=(f, v) \quad \forall v \in D_{*}^{1, \infty}(\Omega),
$$

and

$$
D(u)=F(S)=S\left(1+|S|^{r}\right)^{-\frac{1}{r}} \quad \text { with } \begin{cases}r \in(0,1] & \text { if } d=2, \\ r \in(0,2 / d) & \text { if } d>2 .\end{cases}
$$

Furthermore, the sequence of (uniquely defined) solution pairs $\left(S_{N}, u_{N}\right) \in \Sigma_{N} \times V_{N}, N \geq 1$, generated by (6)-(8), converges to $(S, u)$ in the following sense:

(a) The sequence $\left\{u_{N}\right\}_{N \geq 1}$ converges to $u$ strongly in $\left[L_{\#}^{p}(\Omega)\right]^{d}$ and weakly in $\left[W_{\#}^{1, p}(\Omega)\right]^{d}$ for all $p \in[1, \infty)$;

(b) The sequence $\left\{D\left(u_{N}\right)\right\}_{N \geq 1}$ converges to $D(u)$ weakly in $\left[L_{\#}^{p}(\Omega)\right]^{d \times d}$ for all $p \in[1, \infty)$;

(c) The sequence $\left\{S_{N}\right\}_{N \geq 1}$ converges to $S$ strongly in $\left[L_{\#}^{s}(\Omega)\right]^{d \times d}$ for all values of $s$ in the range $[1, d(1-r) /(d-2))$ for $r \in(0,2 / d)$ when $d>2$, and for all values of $s \in[1, \infty)$ if $r \in(0,1]$ and $d=2$;

(d) The sequence $\left\{D\left(u_{N}\right)\right\}_{N \geq 1}$ converges to $D(u)$ weakly in $\left[W_{\#}^{1,2}(\Omega)\right]^{d \times d}$, and therefore also strongly in $\left[L_{\#}^{p}(\Omega)\right]^{d \times d}$ for all $p \in[1, \infty)$;

(e) If $r \in(0,1 /(d-1)), d \geq 2$, then the sequence $\left\{S_{N}\right\}_{N \geq 1}$ converges to $S$ weakly in $\left[W_{\#}^{1, \theta}(\Omega)\right]^{d \times d}$ for all $\theta \in[1, d(1-r) /(d-r-1))$. 
Proof. The proof consists of two parts. First we shall prove existence of solutions; having done so, we shall proceed to prove uniqueness of the solution.

Existence of solutions. We begin by noting that, thanks to the definition of $\hat{D}_{N}$, we immediately have that, for all $N \geq 1$,

$$
\left\|\hat{D}_{N}\right\|_{L^{\infty}(\Omega)} \leq 1 .
$$

Consequently, denoting by $P_{N}$ the $\left[L_{\#}^{2}(\Omega)\right]^{d \times d}$ orthogonal projector onto $\Sigma_{N}$, we also have that, for all $N \geq 1$,

$$
\left\|D\left(u_{N}\right)\right\|_{L^{p}(\Omega)}=\left\|P_{N} \hat{D}_{N}\right\|_{L^{p}(\Omega)} \leq C_{0}, \quad p \in(1, \infty),
$$

thanks to the stability of the $\left[L_{\#}^{2}(\Omega)\right]^{d \times d}$ orthogonal projector in the $\left[L_{\#}^{p}(\Omega)\right]^{d \times d}$ norm (cf. Canuto, Hussaini, Quarteroni, Zang [4], ineq. (5.1.14) on p.271), where $C_{0}=C_{0}(p)$ signifies a generic positive constant, independent of $N$, whose value may change from one occurrence to another. Thus, by Lemma A.1, also $\left\|u_{N}\right\|_{L^{p}(\Omega)} \leq C_{0}$ for all $N \geq 1$, and any $p \in(1, \infty)$; as $\Omega$ is bounded, also, $\left\|u_{N}\right\|_{L^{1}(\Omega)} \leq C_{0}$ for all $N \geq 1$.

From these bounds, by the compact embedding of $W_{*}^{1, p}(\Omega)$ into $C^{0, \alpha}(\bar{\Omega})$ with $\alpha=1-d / p$, for $p \in(d, \infty)$, we deduce the existence of a function $u \in\left[W_{*}^{1, p}(\Omega)\right]^{d}$ such that (upon extraction of a subsequence, which we shall not denote here and henceforth), as $N \rightarrow \infty$ we have that

$$
\begin{aligned}
u_{N} & \rightarrow u & & \text { weakly in }\left[W_{*}^{1, p}(\Omega)\right]^{d} \text { for all } p \in[1, \infty), \\
u_{N} & \rightarrow u & & \text { strongly in }\left[C^{0, \alpha}(\bar{\Omega})\right]^{d} \text { for all } \alpha \in(0,1), \\
D\left(u_{N}\right) & \rightarrow D(u) & & \text { weakly in }\left[L_{\#}^{p}(\Omega)\right]^{d \times d} \text { for all } p \in[1, \infty), \\
\hat{D}_{N} & \rightarrow D(u) & & \text { weakly in }\left[L_{\#}^{p}(\Omega)\right]^{d \times d} \text { for all } p \in[1, \infty) .
\end{aligned}
$$

To see that $\left\{D\left(u_{N}\right)\right\}_{N \geq 1}$ and $\left\{\hat{D}_{N}\right\}_{N \geq 1}$ converge weakly in $\left[L_{\#}^{p}(\Omega)\right]^{d \times d}$ to the same limit, denote their respective weak limits by $D(u)$ (as in $(15))$ and $\hat{D}$. We will show that $D(u)=\hat{D}$, which will then fully justify the statement (16). Indeed,

$$
(D(u)-\hat{D}, T)=\left(D(u)-D\left(u_{N}\right), T\right)+\left(D\left(u_{N}\right)-\hat{D}_{N}, T\right)+\left(\hat{D}_{N}-\hat{D}, T\right) \quad \forall T \in\left[W_{\#}^{1, p^{\prime}}(\Omega)\right]^{d \times d} .
$$

The first and third term on the right-hand side tend to 0 as $N \rightarrow \infty$ by the definitions of the respective weak limits, so it remains to show that the second term also tends to 0 . Thanks to (8), for any fixed $T \in\left[W_{\#}^{1, p^{\prime}}(\Omega)\right]^{d \times d}$, we have that

$\left(D\left(u_{N}\right)-\hat{D}_{N}, T\right)=\left(D\left(u_{N}\right)-\hat{D}_{N}, T-T_{N}\right) \leq\left\|D\left(u_{N}\right)-\hat{D}_{N}\right\|_{L^{p}(\Omega)}\left\|T-T_{N}\right\|_{L^{p^{\prime}}(\Omega)} \quad \forall T_{N} \in \Sigma_{N}$, and hence, by boundedness in $\left[L_{\#}^{p}(\Omega)\right]^{d \times d}$ of the weakly convergent sequences $\left\{D\left(u_{N}\right)\right\}_{N \geq 1}$ and $\left\{\hat{D}_{N}\right\}_{N \geq 1}$, we have that

$$
\left|\left(D\left(u_{N}\right)-\hat{D}_{N}, T\right)\right| \leq C_{0} \inf _{T_{N} \in \Sigma_{N}}\left\|T-T_{N}\right\|_{L^{p^{\prime}}(\Omega)} \quad \forall T \in\left[W_{\#}^{1, p^{\prime}}(\Omega)\right]^{d \times d} .
$$

As $N \rightarrow \infty$ the right-hand side converges to zero; therefore the same is true of the left-hand side. This proves that $(D(u)-\hat{D}, T)=0$ for all $T \in\left[W_{\#}^{1, p^{\prime}}(\Omega)\right]^{d \times d}$, which by the density of $\left[W_{\#}^{1, p^{\prime}}(\Omega)\right]^{d \times d}$ in $\left[L_{\#}^{p^{\prime}}(\Omega)\right]^{d \times d}$ implies that $D(u)=\hat{D}$, i.e., that $D\left(u_{N}\right)$ and $\hat{D}_{N}$ converge weakly in $\left[L_{\#}^{p}(\Omega)\right]^{d \times d}$ to the same limit, $D(u) \in\left[L_{\#}^{p}(\Omega)\right]^{d \times d}, p \in(1, \infty)$; hence, also for $p=1$. We note also that, by $(12),\left\{\hat{D}_{N}\right\}_{N \geq 1}$ has a subsequence that converges weak-* in $\left[L_{\#}^{\infty}(\Omega)\right]^{d \times d}$ to some $\chi \in\left[L_{\#}^{\infty}(\Omega)\right]^{d \times d}$. By uniqueness of the weak limit, however $\chi=D(u)$; hence, $D(u) \in\left[L_{\#}^{\infty}(\Omega)\right]^{d \times d}$; also, $\int_{\Omega} u(x) \mathrm{d} x=0$. Thus, in view of (14)-(15), we have proved (a) and (b) in the statement of the theorem, as well as that $u \in D_{*}^{1, \infty}(\Omega)$.

In order to prove (c), we take $v_{N}=u_{N}$ in (6), integrate by parts and note (7); thus,

$$
\left(f, u_{N}\right)=\left(S_{N}, D\left(u_{N}\right)\right)=\left(S_{N}, \hat{D}_{N}\right)=\int_{\Omega} \frac{\left|S_{N}(x)\right|^{2}}{\left(1+\left|S_{N}(x)\right|^{r}\right)^{1 / r}} \mathrm{~d} x .
$$


Hence, for all $N \geq 1$,

$$
\int_{\Omega} \frac{\left|S_{N}(x)\right|^{2}}{\left(1+\left|S_{N}(x)\right|^{r}\right)^{1 / r}} \mathrm{~d} x \leq\|f\|_{L^{p^{\prime}}(\Omega)}\left\|u_{N}\right\|_{L^{p}(\Omega)} \leq C_{0}, \quad \frac{1}{p}+\frac{1}{p^{\prime}}=1, \quad 1<p^{\prime} \leq t,
$$

whereby $\left\|S_{N}\right\|_{L^{1}(\Omega)} \leq C_{0}$. This last statement follows by noting that, on the one hand,

$$
\int_{\left\{x \in \Omega:\left|S_{N}(x)\right| \geq 1\right\}}\left|S_{N}(x)\right| \mathrm{d} x \leq 2^{1 / r} \int_{\left\{x \in \Omega:\left|S_{N}(x)\right| \geq 1\right\}} \frac{\left|S_{N}(x)\right|^{2}}{\left(1+\left|S_{N}(x)\right|^{r}\right)^{1 / r}} \mathrm{~d} x \leq C_{0},
$$

(where we have used that $y /\left(1+y^{r}\right)^{1 / r} \geq 1 / 2^{1 / r}$ for all $y \geq 1$ thanks to the fact that $y \in[0, \infty) \mapsto$ $y /\left(1+y^{r}\right)^{1 / r}$ is strictly monotonic increasing $)$, and on the other, by the Cauchy-Schwarz inequality,

$$
\begin{aligned}
\int_{\left\{x \in \Omega:\left|S_{N}(x)\right| \leq 1\right\}}\left|S_{N}(x)\right| \mathrm{d} x \leq & \left(\int_{\left\{x \in \Omega:\left|S_{N}(x)\right| \leq 1\right\}}\left(1+\left|S_{N}(x)\right|^{r}\right)^{1 / r} \mathrm{~d} x\right)^{1 / 2} \\
& \times\left(\int_{\left\{x \in \Omega:\left|S_{N}(x)\right| \leq 1\right\}} \frac{\left|S_{N}(x)\right|^{2}}{\left(1+\left|S_{N}(x)\right|^{r}\right)^{1 / r}} \mathrm{~d} x\right)^{1 / 2} \\
\leq & 2^{1 /(2 r)|\Omega|^{1 / 2}}\left(\int_{\left\{x \in \Omega:\left|S_{N}(x)\right| \leq 1\right\}} \frac{\left|S_{N}(x)\right|^{2}}{\left(1+\left|S_{N}(x)\right|^{r}\right)^{1 / r}} \mathrm{~d} x\right)^{1 / 2} \leq C_{0},
\end{aligned}
$$

whereby, upon adding the bounds on the integrals over the sets $\left\{x \in \Omega:\left|S_{N}(x)\right| \geq 1\right\}$ and $\left\{x \in \Omega:\left|S_{N}(x)\right| \leq 1\right\}$, we have that, for all $N \geq 1$,

$$
\left\|S_{N}\right\|_{L^{1}(\Omega)} \leq C_{0} .
$$

We need bounds on $S_{N}$ in stronger norms in order to be able to pass to the limit as $N \rightarrow \infty$. To this end, we take $v_{N}=-\operatorname{div} D\left(u_{N}\right)$ in (6); note that such a $v_{N}$ belongs to $V_{N}$ and is therefore a legitimate choice of test function in (6). After performing integrations by parts on both the left-hand side and the right-hand side we deduce that, for all $N \geq 1$,

$$
\left(\nabla S_{N}, \nabla D\left(u_{N}\right)\right)=\left(\nabla f, D\left(u_{N}\right)\right) .
$$

Note further that

$$
\left(\nabla S_{N}, \nabla D\left(u_{N}\right)\right)=-\left(\operatorname{div}\left(\nabla S_{N}\right), D\left(u_{N}\right)\right)=-\left(\operatorname{div}\left(\nabla S_{N}\right), \hat{D}_{N}\right)=\left(\nabla S_{N}, \nabla \hat{D}_{N}\right)
$$

and

We thus have that

$$
\left(\nabla f, D\left(u_{N}\right)\right)=\left(P_{N}(\nabla f), D\left(u_{N}\right)\right)=\left(P_{N}(\nabla f), \hat{D}_{N}\right)
$$

$$
\left(\nabla S_{N}, \nabla \hat{D}_{N}\right)=\left(P_{N}(\nabla f), \hat{D}_{N}\right) .
$$

Differentiating the definition of $\hat{D}_{N}$ in (7) and noting that

$$
\nabla S_{N}: \nabla \hat{D}_{N} \geq \frac{\left|\nabla S_{N}\right|^{2}}{\left(1+\left|S_{N}\right|^{r}\right)^{1 / r}}-\frac{\left|S_{N}\right|^{r}\left|\nabla S_{N}\right|^{2}}{\left(1+\left|S_{N}\right|^{r}\right)^{1+1 / r}}=\frac{\left|\nabla S_{N}\right|^{2}}{\left(1+\left|S_{N}\right|^{r}\right)^{1+1 / r}},
$$

we thus have that, for all $N \geq 1$,

$$
\begin{aligned}
\int_{\Omega} \frac{\left|\nabla S_{N}\right|^{2}}{\left(1+\left|S_{N}\right|^{r}\right)^{1+1 / r}} \mathrm{~d} x & \leq\left(P_{N}(\nabla f), \hat{D}_{N}\right) \leq\left\|P_{N}(\nabla f)\right\|_{L^{p}(\Omega)}\left\|\hat{D}_{N}\right\|_{L^{p^{\prime}}(\Omega)} \\
& \leq|\Omega|^{1 / p^{\prime}}\left\|P_{N}(\nabla f)\right\|_{L^{p}(\Omega)} \leq c_{p}|\Omega|^{1 / p^{\prime}}\|\nabla f\|_{L^{p}(\Omega)} \leq C_{0},
\end{aligned}
$$

where in the penultimate inequality we used the stability of the $\left[L_{\#}^{2}(\Omega)\right]^{d \times d}$ projector $P_{N}$ in the $\left[L_{\#}^{p}(\Omega)\right]^{d \times d}$ norm, with $\frac{1}{p}+\frac{1}{p^{\prime}}=1,1<p \leq t$.

It follows from Lemma 1 that

$$
\left(1+y^{r}\right)^{1+1 / r} \leq \max \left(1,2^{1 / r-r}\right)(1+y)^{r+1}, \quad y \geq 0,
$$

and therefore, assuming that $r \neq 1$ (which then implies that $0<r<2 / d$ for all $d \geq 2$ ),

$$
\left\|\nabla\left(1+\left|S_{N}\right|\right)^{\frac{1-r}{2}}\right\|_{L^{2}(\Omega)}^{2} \leq\left(\frac{1-r}{2}\right)^{2} \int_{\Omega} \frac{\left|\nabla S_{N}\right|^{2}}{\left(1+\left|S_{N}\right|\right)^{r+1}} \mathrm{~d} x \leq C_{0} \quad \forall N \geq 1 .
$$


As $0<r<2 / d$, we have by Sobolev embedding that, for all $p$ such that $2<p<2 d /(d-2)$ and $0<r<1-(2 / p)<2 / d$

$$
\int_{\Omega}\left|S_{N}\right|^{p(1-r) / 2} \mathrm{~d} x \leq \int_{\Omega}\left[\left(1+\left|S_{N}\right|\right)^{\frac{1-r}{2}}\right]^{p} \mathrm{~d} x=\left\|\left(1+\left|S_{N}\right|\right)^{\frac{1-r}{2}}\right\|_{L^{p}(\Omega)}^{p} \leq C_{0} \quad \forall N \geq 1,
$$

and the sequence $\left\{S_{N}\right\}_{N \geq 1}$ is therefore bounded in $\left[L_{\#}^{p(1-r) / 2}(\Omega)\right]^{d \times d}$, with $p(1-r) / 2>1$. Thus we can extract a subsequence, which is weakly convergent in $\left[L_{\#}^{p(1-r) / 2}(\Omega)\right]^{d \times d}$; we denote the corresponding weak limit by $S ; S \in\left[L_{\#}^{p(1-r) / 2}(\Omega)\right]^{d \times d}$. As $p$ approaches $2 d /(d-2)$ from below, $1-(2 / p)$ approaches $2 / d$ from below; thus, for any $r$ such that $0<r<2 / d, p$ can be chosen to be arbitrarily close to $2 d /(d-2)$; hence,

$$
S_{N} \stackrel{N \rightarrow \infty}{\rightarrow} S \quad \text { weakly in }\left[L_{\#}^{s}(\Omega)\right]^{d \times d} \text { for all } s \text { such that } 1 \leq s<\frac{d(1-r)}{d-2},\left\{\begin{array}{l}
d \geq 2, \\
0<r<2 / d .
\end{array}\right.
$$

For $0<r<2 / d$, we have that $1<d(1-r) /(d-2)$, and the range of such $s$ is therefore a nonempty half-open interval.

We return to the case of $r=1$, which was excluded in the argument above; this particular case is only of interest when $d=2$ as it corresponds to the upper limit $r=2 / d=1$ in the range of admissible values for $r$. To this end, we take $r=1$ in (18) to deduce that

$$
\left\|\nabla \log \left(1+\left|S_{N}\right|\right)\right\|_{L^{2}(\Omega)}^{2} \leq C_{0} \quad \forall N \geq 1 .
$$

By the continuous embedding of $W_{\#}^{1,2}(\Omega)$ (for $d=2$ ) into the Orlicz space $L_{\#}^{\Psi}(\Omega)$, with $\Psi(t)=$ $\mathrm{e}^{t^{2}}-1$ (as expressed by the Trudinger-Donaldson inequality, for example,) we deduce that, for some constant $\gamma>0$ (independent of $N$ ),

$$
\int_{\Omega} \mathrm{e}^{\gamma\left[\log \left(1+\left|S_{N}\right|\right)\right]^{2}} \mathrm{~d} x \leq C_{0} \quad \forall N \geq 1,
$$

and therefore $\left\{S_{N}\right\}_{N \geq 1}$ is bounded in $L_{\#}^{s}(\Omega)$ for any $s \in[1, \infty)$. Upon extraction of a subsequence, we have weak convergence of $\left\{S_{N}\right\}_{N \geq 1}$ in $\left[L_{\#}^{s}(\Omega)\right]^{d \times d}$ for all $s \in(1, \infty)$, and therefore also for $s=1$. In other words, the following modification of (21) holds:

$$
S_{N} \stackrel{N \rightarrow \infty}{\rightarrow} S \text { weakly in }\left[L_{\#}^{s}(\Omega)\right]^{d \times d} \text { for all } s \text { such that } 1 \leq s<\infty, d=2, r=1 .
$$

We postpone the proof of strong convergence of the sequence $\left\{S_{N}\right\}_{N \geq 1}$ until after we have shown that the pair of functions $(S, u)$ thus identified by the limiting procedure is a weak solution of the problem under consideration; in particular, we shall now show that $(S, D(v))=(f, v)$ for all $v \in D_{*}^{1, \infty}(\Omega)$, that $F(S)=D(u)$, and that $\left\{F\left(S_{N}\right)\right\}_{N \geq 1}$ converges to $F(S)$ weak-* in $\left[L_{\#}^{\infty}(\Omega)\right]^{d \times d}$. The argument is based on Minty's method.

Let $S$ denote the weak limit in $\left[L_{\#}^{1}(\Omega)\right]^{N \times N}$ of the sequence $\left\{S_{N}\right\}_{N \geq 1}$. Hence, for any $v \in$ $\left[C_{*}^{\infty}(\bar{\Omega})\right]^{d}$ and any $v_{N} \in V_{N}$, we have that

$$
\begin{aligned}
\left|\left(S_{N}, D(v)\right)-(f, v)\right| & =\left|\left(S_{N}, D(v)-D\left(v_{N}\right)\right)+\left(f, v_{N}-v\right)\right| \\
& \leq\left(\left\|S_{N}\right\|_{L^{1}(\Omega)}+\|f\|_{L^{1}(\Omega)}\right)\left\|v-v_{N}\right\|_{W^{1, \infty}(\Omega)} .
\end{aligned}
$$

Thus, by (17),

$$
\left|\left(S_{N}, D(v)\right)-(f, v)\right| \leq\left(C_{0}+\|f\|_{L^{1}(\Omega)}\right) \inf _{v_{N} \in V_{N}}\left\|v-v_{N}\right\|_{W^{1, \infty}(\Omega)} \quad \forall v \in\left[C_{*}^{\infty}(\bar{\Omega})\right]^{d} .
$$

By letting $N \rightarrow \infty$ and noting that the right-hand side converges to 0 we deduce, using the weak convergence of $\left\{S_{N}\right\}_{N \geq 1}$ to $S$ in $\left[L_{\#}^{1}(\Omega)\right]^{d \times d}$ that

$$
(S, D(v))=(f, v) \quad \forall v \in\left[C_{*}^{\infty}(\bar{\Omega})\right]^{d} .
$$

Hence, by noting the definition of $D_{*}^{1, \infty}(\Omega)$ and Lemma 4 , we get that

$$
(S, D(v))=(f, v) \quad \forall v \in D_{*}^{1, \infty}(\Omega) .
$$

It remains to show that $D(u)=F(S)$. We begin by observing that $\left\{\hat{D}_{N}\right\}_{N \geq 1}=\left\{F\left(S_{N}\right)\right\}_{N \geq 1}$ is a bounded sequence in $\left[L_{\#}^{\infty}(\Omega)\right]^{d \times d}$. It therefore has a weak-* convergent subsequence, still 
denoted by $\left\{\hat{D}_{N}\right\}_{N \geq 1}$, with limit $\chi \in\left[L_{\#}^{\infty}(\Omega)\right]^{d \times d}$, say. We have already shown in the discussion following equation (16) that $\left\{D\left(u_{N}\right)\right\}_{N \geq 1}$ and $\left\{\hat{D}_{N}\right\}_{N \geq 1}=\left\{F\left(S_{N}\right)\right\}_{N \geq 1}$ possess the same weak limit, $D(u)$; therefore, $\chi=D(u)$; i.e.,

$$
F\left(S_{N}\right) \stackrel{N \rightarrow \infty}{\rightarrow} D(u) \quad \text { weak-* in }\left[L_{\#}^{\infty}(\Omega)\right]^{d \times d} .
$$

We will now show that $D(u)=F(S)$. Note that, thanks to Lemma 2, and equations (7), (8), (6), for any $T \in\left[L_{\#}^{1}(\Omega)\right]^{d \times d}$,

$$
\begin{aligned}
0 & \leq\left(F\left(S_{N}\right)-F(T), S_{N}-T\right)=\left(F\left(S_{N}\right), S_{N}\right)-\left(F\left(S_{N}\right), T\right)-\left(F(T), S_{N}-T\right) \\
& =\left(\hat{D}_{N}, S_{N}\right)-\left(F\left(S_{N}\right), T\right)-\left(F(T), S_{N}-T\right) \\
& =\left(D\left(u_{N}\right), S_{N}\right)-\left(F\left(S_{N}\right), T\right)-\left(F(T), S_{N}-T\right) \\
& =\left(-\operatorname{div} S_{N}, u_{N}\right)-\left(F\left(S_{N}\right), T\right)-\left(F(T), S_{N}-T\right) \\
& =\left(f, u_{N}\right)-\left(F\left(S_{N}\right), T\right)-\left(F(T), S_{N}-T\right) \\
& \rightarrow(f, u)-(\chi, T)-(F(T), S-T) \quad \text { as } N \rightarrow \infty .
\end{aligned}
$$

Thus, by $(23)$ and since $\chi=D(u)$, we have that

$$
0 \leq(S, D(u))-(D(u), T)-(F(T), S-T) \quad \forall T \in\left[L_{\#}^{1}(\Omega)\right]^{d \times d} .
$$

Equivalently,

$$
0 \leq(D(u)-F(T), S-T) \quad \forall T \in\left[L_{\#}^{1}(\Omega)\right]^{d \times d} .
$$

Now consider any $W \in\left[L_{\#}^{1}(\Omega)\right]^{d \times d}$ and take $T=S-\lambda W$, with $\lambda>0$ in (26). Upon division by $\lambda$,

$$
0 \leq(D(u)-F(S-\lambda W), W) \quad \forall W \in\left[L_{\#}^{1}(\Omega)\right]^{d \times d}, \forall \lambda>0 .
$$

Passing to the limit $\lambda \rightarrow 0_{+}$(note that $\lambda \in[0, \infty) \in \mathbb{R} \mapsto(F(S-\lambda W), W) \in \mathbb{R}$ is a continuous function for each fixed $T$ and $W$ ), we have that

$$
0 \leq(D(u)-F(S), W) \quad \forall W \in\left[L_{\#}^{1}(\Omega)\right]^{d \times d} .
$$

Since $\left[L_{\#}^{1}(\Omega)\right]^{d \times d}$ is a linear space, and therefore the last inequality also holds with $W$ replaced by $-W$, we have that

$$
(D(u)-F(S), W)=0 \quad \forall W \in\left[L_{\#}^{1}(\Omega)\right]^{d \times d} .
$$

Thus we have shown that $D(u)=F(S)$, as an equality in $\left[L_{\#}^{\infty}(\Omega)\right]^{d \times d}$, i.e., that

$$
F\left(S_{N}\right) \stackrel{N \rightarrow \infty}{\rightarrow} F(S)=D(u) \quad \text { weak-* in }\left[L_{\#}^{\infty}(\Omega)\right]^{d \times d} .
$$

It remains to prove strong convergence of the sequence $\left\{S_{N}\right\}_{N \geq 1}$. Define

$$
E_{N}(x):=\left(F\left(S_{N}(x)\right)-F(S(x))\right):\left(S_{N}(x)-S(x)\right) .
$$

Then, thanks to Lemma 2 and noting that by the triangle inequality $\left|S_{N}\right| \leq\left|S_{N}-S\right|+|S|$, we obtain the following lower bound on $E_{N}$ :

$$
E_{N}(x) \geq 2^{r-\frac{1}{r}} \frac{\left|S_{N}(x)-S(x)\right|^{2}}{\left(1+\left|S_{N}(x)-S(x)\right|+2|S(x)|\right)^{r+1}}, \quad \text { a.e. } x \in \Omega .
$$

On the other hand, by taking $T=S$ in (25) and noting the fifth line of (25), we have (because $E_{N}$ is nonnegative) and by (23) with $v=u$, that

$$
\begin{aligned}
\int_{\Omega}\left|E_{N}(x)\right| \mathrm{d} x & =\int_{\Omega} E_{N}(x) \mathrm{d} x=\left(f, u_{N}\right)-\left(F\left(S_{N}\right), S\right)-\left(F(S), S_{N}-S\right) \\
& =\left(f, u_{N}-u\right)+\left(F(S), S-S_{N}\right)+\left(D(u)-F\left(S_{N}\right), S\right) \quad \forall N \geq 1 .
\end{aligned}
$$

According to (14), (21) (or (22) if $r=1$ and $d=2$ ) and (24) the right-hand side converges to 0 as $N \rightarrow \infty$; thus, $E_{N} \rightarrow 0$ strongly in $L_{\#}^{1}(\Omega)$. Hence we can extract a subsequence, still denoted by $E_{N}$, which converges to 0 almost everywhere on $\Omega$. The right-hand side of (28) is a nonnegative, continuous, strictly monotonic increasing function of $\left|S_{N}(x)-S(x)\right|$, which vanishes if, and only if, $\left|S_{N}(x)-S(x)\right|=0$. Therefore, $\left|S_{N}(x)-S(x)\right|$ must also converge to 0 almost everywhere on $\Omega$. 
In other words, $\left\{S_{N}\right\}_{N \geq 1}$ converges to $S$ almost everywhere on $\Omega$. By Vitali's theorem we deduce from this, together with (21) (or (22) if $r=1$ and $d=2$ ), that $\left\{S_{N}\right\}_{N \geq 1}$ converges to $S$ strongly in $\left[L^{s}(\Omega)\right]^{d \times d}$ for $1 \leq s<\frac{d(1-r)}{d-2}$ when $d>2$, and for $1 \leq s<\infty$ when $r \in(0,1]$ and $d=2$. That proves part (c).

Next we prove part (d). By (7) and (19) (cf. also (18) for the case of $r=1$ and $d=2$ ), we have that

$$
\int_{\Omega}\left|\nabla \hat{D}_{N}\right|^{2} \mathrm{~d} x \leq \int_{\Omega}\left[\frac{2\left|\nabla S_{N}\right|}{\left(1+\left|S_{N}\right|^{r}\right)^{\frac{1}{r}}}\right]^{2} \mathrm{~d} x=4 \int_{\Omega} \frac{\left|\nabla S_{N}\right|^{2}}{\left(1+\left|S_{N}\right|\right)^{r+1}} \frac{\left(1+\left|S_{N}\right|\right)^{r+1}}{\left(1+\left|S_{N}\right|^{r}\right)^{2 / r}} \mathrm{~d} x \leq C_{0}
$$

for all $N \geq 1$, since the fraction appearing in the integrand as second factor is bounded by 1 for all $r \in(0,1]$ by Lemma 1. As, also, $\left\|\hat{D}_{N}\right\|_{L^{\infty}(\Omega)} \leq 1$, we deduce that $\left\{\hat{D}_{N}\right\}_{N \geq 1}$ is bounded in $\left[W_{\#}^{1,2}(\Omega)\right]^{d \times d}$. Further, by $(8), D\left(u_{N}\right)=P_{N} \hat{D}_{N}$; note also that $P_{N}$ commutes with differentiation and is stable in the $\left[L_{\#}^{2}(\Omega)\right]^{d \times d \times d}$ norm. Thus we have that

$$
\left\|\nabla D\left(u_{N}\right)\right\|_{L^{2}(\Omega)}=\left\|\nabla P_{N} \hat{D}_{N}\right\|_{L^{2}(\Omega)}=\left\|P_{N} \nabla \hat{D}_{N}\right\|_{L^{2}(\Omega)} \leq\left\|\nabla \hat{D}_{N}\right\|_{L^{2}(\Omega)} \leq C_{0} \quad \forall N \geq 1 .
$$

As $\left\{D\left(u_{N}\right)\right\}_{N \geq 1}$ is already known to be bounded in $\left[L_{\#}^{p}(\Omega)\right]^{d \times d}$ for all $p \in[1, \infty)$, (cf. part (a)), we then have that $\left\{D\left(u_{N}\right)\right\}_{N \geq 1}$ is bounded in $\left[W_{\#}^{1,2}(\Omega)\right]^{d \times d}$, just as $\left\{\hat{D}_{N}\right\}_{N \geq 1}$. The statement in part (d) regarding weak convergence of the sequence $\left\{D\left(u_{N}\right)\right\}_{N \geq 1}$ in $\left[W_{\#}^{1,2}(\Omega)\right]^{d \times d}$ then directly follows. The strong convergence is then a consequence of the compact embedding theorem and the fact that $\left\|D\left(u_{N}\right)\right\|_{L^{p}(\Omega)} \leq C_{0}$ for all $p \in[1, \infty)$.

Finally, we prove part (e), now under the more restrictive hypothesis that $0<r<1 /(d-1)$. For any $\theta \in(1,2)$ (to be fixed later on in the argument) note that, thanks to Hölder's inequality (with conjugate exponents $2 / \theta$ and $2 /(2-\theta)$ ) and (19), we have, for any $N \geq 1$,

$$
\begin{aligned}
\int_{\Omega}\left|\nabla S_{N}\right|^{\theta} \mathrm{d} x & =\int_{\Omega}\left[\frac{\left|\nabla S_{N}\right|^{2}}{\left(1+\left|S_{N}\right|\right)^{r+1}}\right]^{\frac{\theta}{2}}\left(1+\left|S_{N}\right|\right)^{\frac{(r+1) \theta}{2}} \mathrm{~d} x \\
& \leq\left[\int_{\Omega} \frac{\left|\nabla S_{N}\right|^{2}}{\left(1+\left|S_{N}\right|\right)^{r+1}} \mathrm{~d} x\right]^{\frac{\theta}{2}}\left[\int_{\Omega}\left(1+\left|S_{N}\right|\right)^{\frac{(r+1) \theta}{2-\theta}} \mathrm{d} x\right]^{1-\frac{\theta}{2}} \\
& \leq C_{0}\left[\int_{\Omega}\left(1+\left|S_{N}\right|\right)^{\frac{(r+1) \theta}{2-\theta}} \mathrm{d} x\right]^{1-\frac{\theta}{2}} .
\end{aligned}
$$

It remains to bound the term in the square brackets on the right-hand side. We have from the bound (20) that

$$
\int_{\Omega}\left(1+\left|S_{N}\right|\right)^{\frac{(1-r) p}{2}} \mathrm{~d} x \leq C_{0} \quad \forall p \in[1,2 d /(d-2)), \quad \forall N \geq 1,
$$

which motivates us to link $\theta$ to $p$ by demanding that

$$
\frac{(r+1) \theta}{2-\theta}=\frac{(1-r) p}{2}
$$

i.e., that

$$
\theta=\frac{2 p(1-r)}{2(r+1)+p(1-r)} .
$$

Trivially, $\theta<2$; in order to ensure that $\theta>1$, we demand that $p>2(r+1) /(1-r)$. Thanks to the assumption that $0<r<1 /(d-1)$, we have that

$$
\frac{2(r+1)}{1-r}<\frac{2 d}{d-2},
$$

and the set of $p$ and $\theta$ that satisfy the requirements that $1 \leq p<2 d /(d-2), 1<\theta<2$, such that the equality (30) holds, is therefore nonempty. With such $\theta$ and $p$, for $0<r<1 /(d-1)$ and $d \geq 2$ fixed, we have that

$$
\int_{\Omega}\left|\nabla S_{N}\right|^{\theta} \mathrm{d} x \leq C_{0} \quad \forall N \geq 1 .
$$


By noting the definition of $\theta$ in terms of $p$ and $r$ and the restriction on the range of $p$, i.e., that $1 \leq p<2 d /(d-2)$, we deduce that $\left\{S_{N}\right\}_{N \geq 1}$ converges weakly in $\left[W^{1, \theta}(\Omega)\right]^{d \times d}$ for all $\theta \in[1, d(1-r) /(d-r-1))$. That completes the proof of (e). that

Uniqueness of the solution. Suppose that $\left(S_{i}, u_{i}\right) \in\left[L^{1}(\Omega)\right]^{d \times d} \times D_{*}^{1, \infty}(\Omega), i=1,2$, are such

$$
\left(S_{i}, D(v)\right)=(f, v) \quad \forall v \in D_{*}^{1, \infty}(\Omega)
$$

with $D\left(u_{i}\right)=F\left(S_{i}\right)=S_{i}\left(1+\left|S_{i}\right|^{r}\right)^{1 / r} \in\left[L_{\#}^{\infty}(\Omega)\right]^{d \times d}$, where $0<r<2 / d$ when $d>2$ or $0<r \leq 1$ when $d=2$. Upon subtracting and taking $v=u_{1}-u_{2}$ (note that this is an admissible choice, since $\left.u_{i} \in D_{*}^{1, \infty}(\Omega), i=1,2\right)$, we have that

$$
\left(S_{1}-S_{2}, D\left(u_{1}\right)-D\left(u_{2}\right)\right)=0 .
$$

On the other hand, $\left(S_{1}-S_{2}, D\left(u_{1}\right)-D\left(u_{2}\right)\right)=\left(S_{1}-S_{2}, F\left(S_{1}\right)-F\left(S_{2}\right)\right)>0$ by Lemma 2 ; the resulting contradiction implies the uniqueness of the solution.

\section{RenORMALIZED SOLUTIONS}

In this section we introduce an alternative notion of solution to problem (3), (4): that of a renormalized solution. The necessity for introducing this notion of solution is associated with the fact that for $r>1$ (if $d=2$ ) or $r \geq 2 / d$ (if $d \geq 3$ ) we are only able to show the validity of (3), (4) up to a set $\mathcal{Z}$ of zero Lebesgue measure, and the potential loss of equality between the left-hand side and the right-hand side of (3) on $\mathcal{Z}$ can be caused by an a priori unknown singular measure concentrated on the set $\mathcal{Z}$. We shall show however that a renormalized solution will coincide with a weak solution provided that $S$ has an improved integrability property. This statement is made more precise in the following theorem.

Theorem 2. Suppose that $f \in\left[W_{*}^{1, t}(\Omega)\right]^{d}$ for some $t>1$, and let $r>0$ be arbitrary; then, there exists a pair $(S, u) \in\left[L_{\#}^{1}(\Omega)\right]^{d \times d} \times D_{*}^{1, \infty}(\Omega)$ such that

$$
D(u)=F(S)=S\left(1+|S|^{r}\right)^{-\frac{1}{r}}
$$

and

$$
(S, D(v))+\langle\chi, D(v)\rangle=(f, v) \quad \forall v \in\left[C_{*}^{1}(\Omega)\right]^{d},
$$

where $\chi \in\left[\mathcal{M}_{\#}(\Omega)\right]^{d \times d}$ is a symmetric periodic Radon measure that is not absolutely continuous with respect to the Lebesgue measure and is supported on a subset of $\Omega$ of zero Lebesgue measure. Moreover, the following energy inequality holds:

$$
(S, D(u)) \leq(f, u) .
$$

Furthermore, the sequence of (uniquely defined) solution pairs $\left(S_{N}, u_{N}\right) \in \Sigma_{N} \times V_{N}, N \geq 1$, generated by (6)-(8), converges to $(S, u)$ in the following sense:

(a) The sequence $\left\{u_{N}\right\}_{N \geq 1}$ converges to $u$ strongly in $\left[L_{*}^{p}(\Omega)\right]^{d}$ and weakly in $\left[W_{*}^{1, p}(\Omega)\right]^{d}$ for all $p \in[1, \infty)$;

(b) The sequence $\left\{D\left(u_{N}\right)\right\}_{N \geq 1}$ converges to $D(u)$ weakly in $\left[L_{\#}^{p}(\Omega)\right]^{d \times d}$ for all $p \in[1, \infty)$;

(c) The sequence $\left\{S_{N}\right\}_{N \geq 1}$ converges to $S+\chi$ weak-* in $\left[\mathcal{M}_{\#}(\Omega)\right]^{d \times d}$;

(d) The sequence $\left\{D\left(u_{N}\right)\right\}_{N \geq 1}$ converges to $D(u)$ strongly in $\left[L_{\#}^{p}(\Omega)\right]^{d \times d}$ for all $p \in[1, \infty)$;

(e) The sequence $\left\{S_{N}\right\}_{N \geq 1}$ converges to $S$ a.e. in $\Omega$.

In addition,

$$
\frac{S}{(1+|S|)^{\frac{r+1}{2}}} \in\left[W_{\#}^{1,2}(\Omega)\right]^{d \times d} \quad \text { and } \quad g(|S|) \in W_{\#}^{1,2}(\Omega) \text { for any } g \in \mathcal{D}(\mathbb{R}) ;
$$

and, for any $g \in \mathcal{D}(\mathbb{R})$, the following renormalized equation holds:

$$
(S, g(|S|) D(v))+(S, \nabla g(|S|) \otimes v)=(f, g(|S|) v) \quad \forall v \in\left[C_{*}^{\infty}(\Omega)\right]^{d} .
$$


Finally, suppose that $(S, u) \in\left[L_{\#}^{1}(\Omega)\right]^{d \times d} \times D_{*}^{1, \infty}(\Omega)$ is a renormalized solution to $(3)-(4)$; i.e., suppose that $(S, u)$ satisfies (34) and (35). If, in addition,

$$
S \in\left[W_{\#}^{1,1}(\Omega)\right]^{d \times d} \quad \text { or } \quad S \in\left[L_{\#}^{r+1}(\Omega)\right]^{d \times d},
$$

then $(S, u)$ is a weak solution to (3)-(4).

Proof. First, using the sequence generated by (6)-(8), we apply the same procedure as in the preceding section to extract a subsequence that we do not relabel such that (a) and (b) hold.

Further, thanks to the boundedness of the sequence $\left\{S_{N}\right\}_{N \geq 1}$ in $\left[L_{\#}^{1}(\Omega)\right]^{d \times d}$, by applying the Banach-Alaoglou theorem and Lebesgue's decomposition theorem, we deduce the existence of a symmetric periodic Radon measure $S \in\left[\mathcal{M}_{\#}(\Omega)\right]^{d \times d}$ that is absolutely continuous with respect to the Lebesgue measure, and of a symmetric periodic Radon measure $\chi \in\left[\mathcal{M}_{\#}(\Omega)\right]^{d \times d}$ that is not absolutely continuous with respect to the Lebesgue measure, such that (a subsequence of) $\left\{S_{N}\right\}_{N \geq 1}$ converges weak-* to $S+\chi$ in $\left[\mathcal{M}_{\#}(\Omega)\right]^{d \times d}$. That proves (c).

Next we shall establish the characterization of $\chi$ stated after (32), followed by the proofs of (e), (d), (33), (34) and (35) (in that order).

Chacon's biting lemma implies the existence of a nondecreasing sequence $\left\{\Omega_{k}\right\}_{k=1}^{\infty}$ of Lebesguemeasurable sets, $\Omega_{k} \subset \Omega_{k+1} \subset \cdots \subset \Omega$, such that $\lim _{k \rightarrow \infty}\left|\Omega \backslash \Omega_{k}\right|=0$, and of a (Lebesgue measurable) function $\hat{S} \in\left[L_{\#}^{1}(\Omega)\right]^{d \times d}$, such that, for each fixed $k \geq 1$, we have

$$
S_{N} \rightarrow \hat{S} \quad \text { weakly in }\left[L^{1}\left(\Omega_{k}\right)\right]^{d \times d} .
$$

In particular, $\hat{S}$ can be assigned a Radon measure, in $\left[\mathcal{M}\left(\Omega_{k}\right)\right]^{d \times d}$, whose Radon-Nikodým derivative is precisely $\hat{S}$; hence the assigned Radon measure is absolutely continuous with respect to the Lebesgue measure. As $\left\{S_{N}\right\}_{N \geq 1}$ converges weak-* to $S+\chi$ in $\left[\mathcal{M}\left(\Omega_{k}\right)\right]^{d \times d}$, for each $k \geq 1$, we deduce by uniqueness of the weak limit that $S+\chi=\hat{S}$ in $\left[\mathcal{M}\left(\Omega_{k}\right)\right]^{d \times d}$, for each $k \geq 1$, with $\hat{S}$ on the right-hand side now understood as an element of $\left[\mathcal{M}\left(\Omega_{k}\right)\right]^{d \times d}$. However $S$ is absolutely continuous with respect to the Lebesgue measure (as is $\hat{S}$ ), while $\chi$ is not absolutely continuous with respect to the Lebesgue measure. Hence, $\chi=0$ on each $\Omega_{k}$, and thus $S=\hat{S} \in L^{1}\left(\Omega_{k}\right)$ for each $k \geq 1$. As $\chi=0$ on each $\Omega_{k}$, and $\left|\Omega \backslash \Omega_{k}\right| \rightarrow 0$, it follows that $\chi$ is supported on subset of $\Omega$ of zero Lebesgue measure. Therefore, $S=\hat{S}$ almost everywhere in $\Omega$ and $S=\hat{S} \in\left[L_{\#}^{1}(\Omega)\right]^{d \times d}$.

Next we prove (e). To do so, we first recall (18), which implies that, for all $N \geq 1$,

$$
\int_{\Omega} \frac{\left|\nabla S_{N}\right|^{2}}{\left(1+\left|S_{N}\right|\right)^{r+1}} \mathrm{~d} x \leq C_{0}
$$

Thus, defining

$$
\begin{aligned}
B_{N} & :=\frac{S_{N}}{\left(1+\left|S_{N}\right|\right)^{r+1}}, \\
a_{N} & :=\frac{1}{\left(1+\left|S_{N}\right|\right)^{r}},
\end{aligned}
$$

it follows that, for all $N \geq 1$,

$$
\left\|B_{N}\right\|_{L^{\infty}(\Omega)}+\left\|a_{N}\right\|_{L^{\infty}(\Omega)}+\int_{\Omega}\left|\nabla B_{N}\right|^{2}+\left|\nabla a_{N}\right|^{2} \mathrm{~d} x \leq 2+C \int_{\Omega} \frac{\left|\nabla S_{N}\right|^{2}}{\left(1+\left|S_{N}\right|\right)^{r+1}} \mathrm{~d} x \leq C,
$$

where the last inequality follows from Hölder's inequality. Therefore, thanks to the compactness of the Sobolev embedding of $W_{\#}^{1,2}(\Omega)$ into $L_{\#}^{1}(\Omega)$, there exist subsequences (not indicated) such that

$$
\begin{aligned}
B_{N} \rightarrow B & \text { strongly in }\left[L_{\#}^{1}(\Omega)\right]^{d \times d}, \\
a_{N} \rightarrow a & \text { strongly in } L_{\#}^{1}(\Omega), \\
B_{N} \rightarrow B & \text { a.e. in } \Omega \\
a_{N} \rightarrow a & \text { a.e. in } \Omega .
\end{aligned}
$$


Moreover, since $\left\{S_{N}\right\}_{N \geq 1}$ is a bounded sequence in $\left[L_{\#}^{1}(\Omega)\right]^{d \times d}$, the nonnegativity of $a_{N}$ implies that

Finally, since

$$
a^{-\frac{1}{r}} \in L_{\#}^{1}(\Omega) \Longrightarrow a>0 \text { a.e. in } \Omega \text {. }
$$

$$
S_{N}=B_{N}\left(a_{N}\right)^{-\frac{r+1}{r}}
$$

the above pointwise convergence result implies that

$$
S_{N} \rightarrow \bar{S} \text { a.e. in } \Omega,
$$

where

$$
\bar{S}:=B a^{-\frac{r+1}{r}},
$$

which is a measurable function that is finite a.e. in $\Omega$. On the other hand, from (37) we have weak convergence to $S$ in $\left[L^{1}\left(\Omega_{k}\right)\right]^{d \times d}$, and hence by Vitali's theorem strong convergence in $\left[L^{1}\left(\Omega_{k}\right)\right]^{d \times d}$; because of the uniqueness of the limit we then obtain that

$$
S_{N} \rightarrow S \text { a.e. in } \Omega_{k} .
$$

Thanks to the properties of the sets $\Omega_{k}$ it then follows that

$$
S_{N} \rightarrow S \text { a.e. in } \Omega \text {. }
$$

Moreover, using Fatou's lemma, we deduce that

$$
\int_{\Omega}|S| \mathrm{d} x \leq \liminf _{N \rightarrow \infty} \int_{\Omega}\left|S_{N}\right| \mathrm{d} x \leq C,
$$

which completes the proof of (e). Then, (d) easily follows from the definition of $D\left(u_{N}\right)$ in terms of $S_{N}$. Similarly, one can obtain the energy inequality (33).

The next part of the proof is devoted to establishing the validity of (34) and (35). Having shown pointwise convergence of the sequence $\left\{S_{N}\right\}_{N \geq 1}$, we deduce from the above a priori bounds that

$$
\begin{array}{cc}
\frac{\nabla S_{N}}{\left(1+\left|S_{N}\right|\right)^{\frac{r+1}{2}}} \rightarrow \frac{\nabla S}{(1+|S|)^{\frac{r+1}{2}}} & \text { weakly in }\left[L_{\#}^{2}(\Omega)\right]^{d \times d \times d}, \\
\frac{S_{N}}{\left(1+\left|S_{N}\right|\right)^{r+1}} \rightarrow \frac{S}{(1+|S|)^{r+1}} & \text { weakly in }\left[W_{\#}^{1,2}(\Omega)\right]^{d \times d}, \\
\frac{1}{\left(1+\left|S_{N}\right|\right)^{r}} \rightarrow \frac{1}{(1+|S|)^{r}} & \text { weakly in } W_{\#}^{1,2}(\Omega) .
\end{array}
$$

Now, let $g \in C_{0}^{\infty}(\mathbb{R})$ be arbitrary. Then, the pointwise convergence of $S_{N}$ implies that

$$
g\left(\left|S_{N}\right|\right) \rightarrow g(|S|) \quad \text { strongly in } L_{\#}^{p}(\Omega) \text { for all } p \in[1, \infty) .
$$

Moreover, it also follows from (38) that

$$
g\left(\left|S_{N}\right|\right) \rightarrow g(|S|) \quad \text { weakly in } W_{\#}^{1,2}(\Omega) .
$$

In addition, as $g$ has compact support, Lebesgue's dominated convergence theorem implies that

$$
g\left(\left|S_{N}\right|\right) S_{N} \rightarrow g(|S|) S \quad \text { strongly in }\left[L_{\#}^{p}(\Omega)\right]^{d \times d} \text { for all } p \in[1, \infty) .
$$

Finally, using (38) we have that, for all $N \geq 1$,

$$
\int_{\Omega}\left|\nabla\left(g\left(\left|S_{N}\right|\right) S_{N}\right)\right|^{2} \mathrm{~d} x \leq C \int_{\Omega \cap\left\{x ;\left|S_{N}\right| \in \operatorname{supp} g\right\}}\left|\nabla S_{N}\right|^{2} \mathrm{~d} x \leq C .
$$

By combining this with $(40)$ and using the reflexivity of $\left[W_{\#}^{1,2}(\Omega)\right]^{d \times d}$ we deduce that

$$
g\left(\left|S_{N}\right|\right) S_{N} \rightarrow g(|S|) S \quad \text { weakly in }\left[W_{\#}^{1,2}(\Omega)\right]^{d \times d} .
$$

Hence, setting $v_{N}:=P_{N}\left(g\left(\left|S_{N}\right|\right) v\right)$ in (6) with arbitrary $v \in C_{\#}^{1}(\Omega)$, we obtain the following identity

$$
\int_{\Omega} g\left(\left|S_{N}\right|\right) S_{N}: \nabla v \mathrm{~d} x+\int_{\Omega} S_{N}:\left(\nabla g\left(\left|S_{N}\right|\right) \otimes v\right) \mathrm{d} x=\int_{\Omega} P_{N} f \cdot g\left(\left|S_{N}\right|\right) v \mathrm{~d} x
$$


Finally, we let $N \rightarrow \infty$ in (42) to obtain (35). Indeed, for the term on the right-hand side we use (39). For the first term on the left-hand side we use (40) and the second term on the left-hand side can be handled with the help of (41).

It remains to verify that (36) leads to the implication renormalized solution $\Longrightarrow$ weak solution. To this end, we consider (35) with $g_{k} \in \mathcal{D}(\mathbb{R})$ defined, for $k \in \mathbb{N}$, in the following way: $g_{k}(s)=$ $g_{k}(-s), g_{k}(s)=1$ for $s \in[0, k], g_{k}(s)=0$ for $s \in[2 k,+\infty)$ and $\left|g_{k}^{\prime}(s)\right| \leq \frac{2}{k}$ for $s \in(k, 2 k)$. Then, letting $k$ tend to $\infty$ in (35), we shall arrive at $(S, D(v))=(f, v)$ for all $v \in\left[C_{*}^{\infty}(\Omega)\right]^{d}$ provided that we can show that (36) implies that

$$
\limsup _{k \rightarrow \infty}\left(S, \nabla g_{k}(|S|) \otimes v\right)=0 .
$$

However,

$$
\left|\left(S, \nabla g_{k}(|S|) \otimes v\right)\right| \leq C \int_{\Omega} g_{k}^{\prime}(|S|)|S||\nabla S| \mathrm{d} x \leq C \int_{\{x \in \Omega: k \leq|S(x)| \leq 2 k\}} \frac{2 k}{k}|\nabla S| \mathrm{d} x,
$$

and the last term vanishes, as $k \rightarrow \infty$, if $S \in\left[W_{\#}^{1,1}(\Omega)\right]^{d \times d}$. If on the other hand $S \in\left[L_{\#}^{r+1}(\Omega)\right]^{d \times d}$, then we use (38) to deduce that

$$
\left|\left(S, \nabla g_{k}(|S|) \otimes v\right)\right| \leq C \int_{\{x \in \Omega: k \leq|S(x)| \leq 2 k\}} \frac{|\nabla S|}{(1+|S|)^{\frac{r+1}{2}}}(1+|S|)^{\frac{r+1}{2}} \mathrm{~d} x \rightarrow 0 \text { as } k \rightarrow \infty .
$$

That completes the proof.

\section{Conclusion}

This study contributes to the analysis of boundary-value problems describing the static state of implicitly constituted elastic solids, as formulated in (1). In this generality, there are no results known to the authors that are concerned with the analysis of the problem (1) and which involve the left Cauchy-Green deformation tensor $B$. The only results, obtained recently (some of them are presented in herein), concern a version of problem (1) where the tensor $B$ is replaced by the linearized strain $D(u)=\varepsilon(u)$. Regarding this setting, the state of the art concerning the analysis of the relevant boundary-value problems is the following:

(a) Consider $-\operatorname{div} T=0$ with (2) in $\Omega$ and a prescribed traction over the whole boundary (i.e., $T n=g$ on the boundary) in a special geometric setting (a cylinder $O \times(-\infty, \infty)$ with a planar cross-section $O \subset \mathbb{R}^{2}$ ) for a special deformation called anti-plane strain. One is then allowed to introduce the Airy stress function $U: O \rightarrow \mathbb{R}$ and the whole problem reduces to a scalar Dirichlet problem

$$
-\operatorname{div}\left(\frac{\nabla U}{\left(1+|\nabla U|^{r}\right)^{1 / r}}\right)=0 \quad \text { in } O, \quad U=U_{0} \quad \text { on } \partial O .
$$

Note that the Neumann-type boundary condition for the original problem leads to a nonhomogeneous Dirichlet boundary condition for the Airy stress function over the entire boundary $\partial O$. In [2], the following results are established: if $\Omega$ is convex, then there is a unique weak solution to the problem for all $r \in(0, \infty)$. If $\Omega$ is nonconvex (such as a $\mathrm{V}$-notch, for example,) with constant Dirichlet data on nonconvex parts of the boundary, the existence of a unique weak solution is established for all $r \in(0,2)$. Since it is known that there is a nonconvex set $\Omega$ such that a weak solution to (6) for $r=2$ does not exist, the results concerning nonconvex (as well as convex) domains seem to be sharp.

(b) In this study, we have considered the system of partial differential equations $-\operatorname{div} T=f$ with (2) in any number of space dimensions; we have however confined ourselves to the spatially periodic setting. Relying on a constructive approximation method, based on a Fourier spectral method, we established the existence of a weak solution and its uniqueness for $r \in(0,2 / d)$. We have further introduced the concept of renormalized solution, proved its existence for any $r \in(0, \infty)$, and we stated conditions on $S$ that suffice for one to deduce that a renormalized solution is in fact a weak solution. 
(c) In general situations, still within the framework involving the linearized strain, most of the analytical problems concerning existence, uniqueness and stability of solutions are open. The only exception is the existence of a weak solution to (1) with nonhomogeneous Dirichlet data, i.e., $\Gamma_{N}=\emptyset$, for $r \in(0,1 / d)$; the proof of existence of a weak solution in this case will be presented in a forthcoming paper, see [1]. There are no results we are aware of concerning the existence of renormalized solutions on general bounded domains, with either Dirichlet, or Neumann, or mixed boundary condition on the displacement $u$.

\section{REFERENCES}

[1] M. Bulíček, J. Málek, K. R. Rajagopal, And E. SüLi, On elastic solids with limiting small strain: modelling and analysis. Submitted for publication, 2014.

[2] M. BulíčEK, J. Málek, K. R. Rajagopal, and J. Walton, Existence of solutions for the anti-plane stress for a new class of "strain-limiting" elastic bodies. Submitted for publication, 2014.

[3] R. Bustamante and K. R. Rajagopal, Solutions of some simple boundary value problems within the context of a new class of elastic materials, International Journal of Non-Linear Mechanics, 46 (2011), pp. 376-386.

[4] C. Canuto, M. Hussaini, A. Quarteroni, And T. Zang, Spectral Methods: Fundamentals in Single Domains, Scientific Computation. Berlin: Springer. xxiii, 563 p., 2006.

[5] A. D. Freed, Soft Solids - A primer to the theoretical mechanics of materials, Create Space Independent Publishing Platform, Norh Charleston, South Carolina, 2012.

[6] A. D. Freed and D. R. Einstein, An implicit elastic theory for lung parenchyma, Internat. J. Engrg. Sci., 62 (2013), pp. 31-47.

[7] V. Girault and P.-A. Raviart, Finite Element Approximation of the Navier-Stokes Equations, vol. 749 of Lecture Notes in Mathematics, Springer-Verlag, 1979.

[8] — Finite Element Methods for Navier-Stokes Equations, vol. 5 of Springer Ser. Comp. Math., SpringerVerlag, 1986.

[9] V. Kulvait, J. Málek, and K. Rajagopal, Anti-plane stress state of a plate with a V-notch for a new class of elastic solids, Int. J. Fract., 179 (2013), pp. 59-73.

[10] A. Ortiz, R. Bustamante, and K. R. Rajagopal, A numerical study of a plate with a hole for a new class of elastic bodies, Acta Mech., 223 (2012), pp. 1971-1981.

[11] K. R. Rajagopal, On implicit constitutive theories, Appl. Math., 48 (2003), pp. 279-319.

[12] - Elasticity of elasticity, Zeitschrift fur Angewandte Math Phys, 58 (2007), pp. 309-417.

[13] — Conspectus of concepts of elasticity, Math. Mech. Solids, 16 (2011), pp. 536-562.

[14] - Non-linear elastic bodies exhibiting limiting small strain, Math. Mech. Solids, 16 (2011), pp. 122-139.

[15] — On a new class of models in elasticity, J. Math. Comp. Appl., 15 (2011), pp. 506-528.

[16] — On the nonlinear elastic response of bodies in the small strain range, Acta Mechanica, (2013), pp. 1-9.

[17] K. R. Rajagopal and A. R. SRinivasa, On the response of non-dissipative solids, Proc. Roy. Soc. London A, 463 (2007), pp. 357-367.

[18] — On a class of non-dissipative materials that are not hyperelastic, Proc. Roy. Soc. London A, 465 (2009), pp. 493-500.

[19] K. R. Rajagopal and J. Walton, Modeling fracture in the context of strain-limiting theory of elasticity, Int. J. Fract., 169 (2011), pp. 39-48.

[20] H.-J. Schmeisser AND H. Triebel, Topics in Fourier analysis and function spaces, A Wiley-Interscience Publication, John Wiley \& Sons Ltd., Chichester, 1987.

[21] E. M. Stein and G. Weiss, Introduction to Fourier analysis on Euclidean spaces, Princeton University Press, Princeton, N.J., 1971. Princeton Mathematical Series, No. 32.

\section{APPENDIX}

The purpose of this Appendix is to prove some auxiliary results, which are required in the arguments in the main body of the paper. In particular, we shall prove the inf-sup condition (5). We begin with the statement of Korn's inequality in the $L^{p}$ norm, whose proof is also included for the sake of completeness, as we were unable to find it in the literature in the context of periodic boundary conditions. For $p=2$ we explicitly calculate (an upper bound on) the constant in Korn's inequality in the $L^{2}$ norm, which then allows us to specify (a lower bound on) the constant $c_{\text {inf-sup }}$ appearing in the inf-sup condition (5).

Lemma A.1 (Korn's inequality in $\left.L^{p}\right)$. Let $p \in(1, \infty), d \geq 2$ and $\Omega:=(0,2 \pi)^{d}$. There exists a positive constant $c_{p}$ such that the following inequalities hold:

$$
\|\nabla v\|_{L^{p}(\Omega)} \leq c_{p}\left(\|D(v)\|_{L^{p}(\Omega)}+\|\operatorname{div} v\|_{L^{p}(\Omega)}\right) \quad \forall v \in\left[W_{*}^{1, p}(\Omega)\right]^{d},
$$


and, hence, also, with a possibly different constant $c_{p}$,

$$
\|\nabla v\|_{L^{p}(\Omega)} \leq c_{p}\|D(v)\|_{L^{p}(v)} \quad \forall v \in\left[W_{*}^{1, p}(\Omega)\right]^{d} .
$$

Let, further, $D^{\operatorname{dev}}(v):=D(v)-\frac{1}{d}(\operatorname{div} v) \mathrm{I}$ denote the deviatoric part of $D(v)$, where $\mathrm{I}$ is the identity matrix in $\mathbb{R}^{d \times d}$; then, there exists a positive constant $c_{p}$ such that

$$
\|\nabla v\|_{L^{p}(\Omega)} \leq c_{p}\left\|D^{\operatorname{dev}}(v)\right\|_{L^{p}(\Omega)} \quad \forall v \in\left[W_{*}^{1, p}(\Omega)\right]^{d} .
$$

Besides being dependent on $p$, the constant $c_{p}$ (whose specific value may change from one line to the next) also depends on $d$, but we do not explicitly indicate that. In each case, the left-hand side of the inequality can be further bounded below by $C_{p}\|v\|_{W^{1, p}(\Omega)}$, where $C_{p}$ is another positive constant dependent on $p$, but independent of $v$.

Proof. The statement in the penultimate sentence of the lemma is an immediate consequence of Poincaré's inequality for functions $v \in\left[W_{*}^{1, p}(\Omega)\right]^{d}$ (which, by definition, have zero integral over $\Omega)$; viz.,

$$
C_{p}\|v\|_{W^{1, p}(\Omega)} \leq\|\nabla v\|_{L^{p}(\Omega)} \quad \forall v \in W_{*}^{1, p}(\Omega) .
$$

The second stated inequality is an immediate consequence of the first inequality, based on the following argument: $\operatorname{div} v=\operatorname{tr} D(v)$; hence, by the Cauchy-Schwarz inequality for matrices,

$$
|\operatorname{div} v|=|\operatorname{tr} D(v)|=|\mathrm{I}: D(v)| \leq|\mathrm{I}||D(v)|=d^{1 / 2}|D(v)|,
$$

and thus, also $\|\operatorname{div} v\|_{L^{p}(\Omega)} \leq d^{1 / 2}\|D(v)\|_{L^{p}(\Omega)}$, so the second stated inequality is implied by the first.

In order to prove the two remaining inequalities we proceed as follows. As $\left[C_{*}^{\infty}(\bar{\Omega})\right]^{d}$ is, by definition, dense in $\left[W_{*}^{1, p}(\Omega)\right]^{d}$ for $p \in(1, \infty)$, it suffices to prove the inequalities for $v \in\left[C_{*}^{\infty}(\bar{\Omega})\right]^{d}$. For any such smooth $v$, we have that

$$
\Delta v=2 \operatorname{div} D(v)-\nabla(\operatorname{div} v) .
$$

Because $\Delta$ has a well-defined inverse when considered as a mapping from $\left[C_{*}^{\infty}(\Omega)\right]^{d}$ into itself, we apply $\Delta^{-1}$ to both sides of the last equality and we then apply the gradient operator $\nabla$ to both sides of the resulting equality, which then yields

$$
\nabla v=2\left(\nabla \Delta^{-1} \operatorname{div} D(v)\right)-\left(\nabla \Delta^{-1} \nabla \operatorname{div} v\right) .
$$

Here, $\nabla v$ is understood to mean the $d \times d$ matrix whose $(i, j)$ entry is $(\nabla v)_{i j}=\frac{\partial}{\partial x_{i}} v_{j}$ for $i, j=$ $1, \ldots, d$. Let us also define

$$
(\mathcal{F} v)(k):=\hat{v}(k)=\frac{1}{(2 \pi)^{d}} \int_{\Omega} v(x) \mathrm{e}^{-\imath k \cdot x} \mathrm{~d} x
$$

so that $v=\mathcal{F}^{-1} \mathcal{F} v$, with

$$
\left(\mathcal{F}^{-1} \mathcal{F} v\right)(x)=\left(\mathcal{F}^{-1} \hat{v}\right)(x)=\sum_{k \in \mathbb{Z}^{d}} \hat{v}(k) \mathrm{e}^{\imath k \cdot x} .
$$

When applied to matrix-functions, the transforms $\mathcal{F}$ and $\mathcal{F}^{-1}$ are understood to be acting componentwise; thus, for example, $\mathcal{F} M$ for a matrix function $M=\left(m_{i j}\right)_{i, j=1}^{d}$ means a matrix whose $(i, j)$ entry is $\mathcal{F} m_{i j}$. A straightforward calculation yields that

$$
\begin{aligned}
\nabla v & =\mathcal{F}^{-1} \mathcal{F}(\nabla v)=2 \mathcal{F}^{-1}\left[\mathcal{F}\left(\nabla \Delta^{-1} \operatorname{div} D(v)\right)\right]-\mathcal{F}^{-1}\left[\mathcal{F}\left(\nabla \Delta^{-1} \nabla \operatorname{div} v\right)\right] \\
& =2 \mathcal{F}^{-1}\left[\frac{k \otimes k}{|k|^{2}} \mathcal{F}(D(v))\right]-\mathcal{F}^{-1}\left[\frac{k \otimes k}{|k|^{2}} \mathcal{F}(\operatorname{div} v)\right] .
\end{aligned}
$$

Here, in the first square bracket we have the product of the $d \times d$ matrix $(k \otimes k) /|k|^{2}$ with the matrix $\mathcal{F}(D(v))$, with $\mathcal{F}$ being applied component-by-component to the entries of the $d \times d$ matrix $D(v)$; while in the second square bracket we have the $d \times d$ matrix $(k \otimes k) /|k|^{2}$ post-multiplied by the scalar $\mathcal{F}(\operatorname{div} v)$. 
Hence,

$$
\begin{aligned}
\|\nabla v\|_{L^{p}(\Omega)} & \leq 2\left\|\mathcal{F}^{-1}\left[\frac{k \otimes k}{|k|^{2}} \mathcal{F}(D(v))\right]\right\|_{L^{p}(\Omega)}+\left\|\mathcal{F}^{-1}\left[\frac{k \otimes k}{|k|^{2}} \mathcal{F}(\operatorname{div} v)\right]\right\|_{L^{p}(\Omega)} \\
& \leq c_{p}\left(\|D(v)\|_{L^{p}(\Omega)}+\|\operatorname{div} v\|_{L^{p}(\Omega)}\right)
\end{aligned}
$$

where in the transition to the last line we used that, thanks to Lizorkin's multiplier theorem, $\xi \in \mathbb{R}^{d} \backslash\{0\} \mapsto \frac{\xi_{i} \xi_{m}}{|\xi|^{2}} \in \mathbb{R}$ is a Fourier multiplier in $L^{p}\left(\mathbb{R}^{d}\right), 1<p<\infty$, and thus by De Leeuw's transference theorem (cf. Theorem 3.8 on p.260 of Stein \& Weiss [21], or Theorem 3.4.2 and Remark 3.4.4 in Schmeisser \& Triebel [20]), its restriction to $\mathbb{Z}^{d} \backslash\{0\}$, i.e., $k \in \mathbb{Z}^{d} \backslash\{0\} \mapsto \frac{k_{i} k_{m}}{|k|^{2}} \in \mathbb{R}$ is a Fourier multiplier in $L_{\#}^{p}(\Omega)$ (and hence in $L_{*}^{p}(\Omega)$ ), for all $i, m=1, \ldots, d$. Since Lizorkin's multiplier theorem is usually formulated for $\mathbb{R}$-valued functions while here we are working with $\mathbb{R}^{d \times d}$-valued functions, we shall provide the details of the calculation for the first summand in the penultimate line above, but will then omit the details of similar subsequent calculations. It has to be borne in mind that the meaning of the $L^{p}$ norm of a certain matrix function, say, $G: x \mapsto\left(G_{i j}(x)\right)_{i, j=1}^{d}$ is that we take the standard $L^{p}$ norm for scalar-valued functions of the matrix norm $|G|$ of $G$. In our case,

$$
G_{i j}=\left(\mathcal{F}^{-1}\left[\frac{k \otimes k}{|k|^{2}} \mathcal{F}(D(v))\right]\right)_{i j}=\sum_{m=1}^{d} \mathcal{F}^{-1}\left[\frac{k_{i} k_{m}}{|k|^{2}} \mathcal{F}\left((D(v))_{m j}\right)\right] .
$$

Now, $|G| \leq \sum_{i, j=1}^{d}\left|G_{i j}\right|$, and therefore

$$
\|G\|_{L^{p}(\Omega)} \leq \sum_{i, j=1}^{d} \sum_{m=1}^{d}\left\|\mathcal{F}^{-1}\left[\frac{k_{i} k_{m}}{|k|^{2}} \mathcal{F}\left((D(v))_{m j}\right)\right]\right\|_{L^{p}(\Omega)} .
$$

We then apply a combination of Lizorkin's theorem and De Leeuw's theorem to each of the $d^{3}$ summands, resulting in

$$
\|G\|_{L^{p}(\Omega)} \leq \sum_{i, j=1}^{d} \sum_{m=1}^{d} m_{p}\left(\frac{k_{i} k_{m}}{|k|^{2}}\right)\left\|(D(v))_{m j}\right\|_{L^{p}(\Omega)},
$$

where $m_{p}(\cdot)$ is the multiplier norm in $L^{p}(\Omega)$. By letting $c_{p}:=\max _{i, m=1, \ldots, d} m_{p}\left(\frac{k_{i} k_{m}}{|k|^{2}}\right)$ and noting that, for each fixed $m, j \in\{1, \ldots, d\},\left|(D(v))_{m j}\right| \leq|D(v)|$ and therefore $\left\|(D(v))_{m j}\right\|_{L^{p}(\Omega)} \leq$ $\||D(v)|\|_{L^{p}(\Omega)}=\|D(v)\|_{L^{p}(\Omega)}$, we have that

$$
\|G\|_{L^{p}(\Omega)} \leq d^{3} c_{p}\|D(v)\|_{L^{p}(\Omega)},
$$

and hence

$$
\left\|\mathcal{F}^{-1}\left[\frac{k \otimes k}{|k|^{2}} \mathcal{F}(D(v))\right]\right\|_{L^{p}(\Omega)} \leq d^{3} c_{p}\|D(v)\|_{L^{p}(\Omega)}
$$

Similarly,

$$
\left\|\mathcal{F}^{-1}\left[\frac{k \otimes k}{|k|^{2}} \mathcal{F}(\operatorname{div} v)\right]\right\|_{L^{p}(\Omega)} \leq \sum_{i, j=1}^{d} m_{p}\left(\frac{k_{i} k_{j}}{|k|^{2}}\right)\|\operatorname{div} v\|_{L^{p}(\Omega)} \leq d^{2} c_{p}\|\operatorname{div} v\|_{L^{p}(\Omega)} .
$$

The factors $d^{3}$ and $d^{2}$ are then absorbed into the symbol $c_{p}$, without further explicit indication of the dependence of $c_{p}$ on $d$. This then proves the first stated inequality.

It remains to prove the third inequality in the statement of the lemma. To this end, we note that

$$
\begin{aligned}
\operatorname{div} D^{\operatorname{dev}}(v) & =\operatorname{div} D(v)-\frac{1}{d} \operatorname{div}((\operatorname{div} v) \mathrm{I}) \\
& =\frac{1}{2} \Delta v+\frac{1}{2} \nabla \operatorname{div} v-\frac{1}{d} \nabla \operatorname{div} v .
\end{aligned}
$$


Hence,

$$
\operatorname{div} \operatorname{div} D^{\operatorname{dev}}(v)=\frac{1}{2} \Delta \operatorname{div} v+\frac{1}{2} \operatorname{div}(\nabla \operatorname{div} v)-\frac{1}{d} \operatorname{div}(\nabla(\operatorname{div} v))=\frac{d-1}{d} \Delta \operatorname{div} v,
$$

whereby

$$
\operatorname{div} v=\frac{d}{d-1} \Delta^{-1} \operatorname{div} \operatorname{div} D^{\operatorname{dev}}(v) .
$$

Using this identity in the transition from the second to the third line in the chain of equalities below, and the definition of $D^{\operatorname{dev}}(v)$ in the transition from the first to the second line yields:

$$
\begin{aligned}
\Delta v & =2 \operatorname{div} D(v)-\nabla \operatorname{div} v \\
& =2 \operatorname{div}\left[D^{\operatorname{dev}}(v)+\frac{1}{d}(\operatorname{div} v) \mathrm{I}\right]-\nabla(\operatorname{div} v) \\
& =2 \operatorname{div} D^{\operatorname{dev}}(v)+\frac{2}{d} \operatorname{div}\left[\frac{d}{d-1}\left(\Delta^{-1} \operatorname{div} \operatorname{div} D^{\operatorname{dev}}(v)\right) \mathrm{I}\right]-\nabla\left[\frac{d}{d-1} \Delta^{-1} \operatorname{div} \operatorname{div} D^{\operatorname{dev}}(v)\right],
\end{aligned}
$$

and therefore, by applying $\Delta^{-1}$ to both sides, and then $\nabla$ to both sides of the resulting equality, we have that

$$
\begin{gathered}
\nabla v=2 \nabla \Delta^{-1} \operatorname{div} D^{\operatorname{dev}}(v)+\frac{2}{d-1} \nabla \Delta^{-1} \operatorname{div}\left[\left(\Delta^{-1} \operatorname{div} \operatorname{div} D^{\operatorname{dev}}(v)\right) \mathrm{I}\right] \\
-\frac{d}{d-1} \nabla \Delta^{-1} \nabla\left[\Delta^{-1} \operatorname{div} \operatorname{div} D^{\operatorname{dev}}(v)\right] .
\end{gathered}
$$

Thus,

$$
\nabla v=\mathcal{F}^{-1} \mathcal{F}(\nabla v)=2 \mathcal{F}^{-1}\left[\frac{k \otimes k}{|k|^{2}} \mathcal{F}\left(D^{\mathrm{dev}}(v)\right)\right]+\frac{2-d}{d-1} \mathcal{F}^{-1}\left[\frac{k \otimes k}{|k|^{2}}\left(\frac{k \otimes k}{|k|^{2}}: \mathcal{F}\left(D^{\mathrm{dev}}(v)\right)\right)\right]
$$

which then implies, with $c_{p}:=\max _{i, j=1, \ldots, d} m_{p}\left(\frac{k_{i} k_{j}}{|k|^{2}}\right)$ and recalling that $d \geq 2$, that

$$
\begin{aligned}
\|\nabla v\|_{L^{p}(\Omega)} \leq & 2\left\|\mathcal{F}^{-1}\left[\frac{k \otimes k}{|k|^{2}} \mathcal{F}\left(D^{\operatorname{dev}}(v)\right)\right]\right\|_{L^{p}(\Omega)} \\
& +\frac{d-2}{d-1}\left\|\mathcal{F}^{-1}\left[\frac{k \otimes k}{|k|^{2}}\left(\frac{k \otimes k}{|k|^{2}}: \mathcal{F}\left(D^{\operatorname{dev}}(v)\right)\right)\right]\right\|_{L^{p}(\Omega)} \\
= & 2\left\|\mathcal{F}^{-1}\left[\frac{k \otimes k}{|k|^{2}} \mathcal{F}\left(D^{\operatorname{dev}}(v)\right)\right]\right\| \|_{L^{p}(\Omega)} \\
& +\frac{d-2}{d-1}\left\|\mathcal{F}^{-1}\left[\frac{k \otimes k}{|k|^{2}} \mathcal{F}\left[\mathcal{F}^{-1}\left(\frac{k \otimes k}{|k|^{2}}: \mathcal{F}\left(D^{\operatorname{dev}}(v)\right)\right)\right]\right]\right\|_{L^{p}(\Omega)} \\
\leq & 2 d^{3} c_{p}\left\|D^{\operatorname{dev}}(v)\right\|_{L^{p}(\Omega)}+\frac{d-2}{d-1} d^{2} c_{p}\left\|\mathcal{F}^{-1}\left(\frac{k \otimes k}{|k|^{2}}: \mathcal{F}\left(D^{\operatorname{dev}}(v)\right)\right)\right\|_{L^{p}(\Omega)} \\
\leq & 2 d^{3} c_{p}\left\|D^{\operatorname{dev}}(v)\right\|_{L^{p}(\Omega)}+\frac{d-2}{d-1} d^{4} c_{p}\left\|D^{\operatorname{dev}}(v)\right\|_{L^{p}(\Omega)},
\end{aligned}
$$

where in the transition to the penultimate line, and then again in the passage to the last line, we used that $k \in \mathbb{Z}^{d} \backslash\{0\} \mapsto k_{i} k_{j} /|k|^{2} \in \mathbb{R}$ is a Fourier multiplier in $L_{*}^{p}(\Omega)$ for all $i, j=1, \ldots, d$ and all $p \in(1, \infty)$. After merging the two terms on the right-hand side in the last displayed line, and absorbing dependence on $d$ into our notation for the constant $c_{p}$, we thus arrive at the third stated inequality.

We shall now consider Lemma A.1 in the special case when $p=2$, and will provide an elementary proof, which will allow us to explicitly compute the constant $c_{p}$ in Korn's inequality stated in Lemma A.1 for $p=2$, and thereby also the constant $c_{\text {inf-sup }}$ appearing in the inf-sup condition (5).

Lemma A.2 (Korn's inequality in $L^{2}$ ). We have that

$$
\|v\|_{L^{2}(\Omega)} \leq \sqrt{2}\|D(v)\|_{L^{2}(\Omega)} \quad \forall v \in\left[W_{*}^{1,2}(\Omega)\right]^{d}
$$


and

$$
\|v\|_{W^{1,2}(\Omega)} \leq 2\|D(v)\|_{L^{2}(\Omega)} \quad \forall v \in\left[W_{*}^{1,2}(\Omega)\right]^{d} .
$$

Proof. Let $v \in\left[C_{*}^{\infty}(\bar{\Omega})\right]^{d}$. Again, we shall use that the function $v$ then has the Fourier series expansion

$$
v(x)=\sum_{k \in \mathbb{Z}^{d} \backslash\{0\}} \hat{v}(k) \mathrm{e}^{i k \cdot x}, \quad \text { where } \quad \hat{v}(k):=\frac{1}{(2 \pi)^{d}} \int_{\Omega} v(x) \mathrm{e}^{-i k \cdot x} \mathrm{~d} x .
$$

By partial integration in the transition from the third to the fourth line below, dropping the nonnegative term $\|\operatorname{div} v\|_{L^{2}(\Omega)}^{2}$ and using Parseval's identity in the transition from the fourth to the fifth line, and again in the last line, we have that

$$
\begin{aligned}
\|D(v)\|_{L^{2}(\Omega)}^{2} & =\frac{1}{4} \sum_{i, j=1}^{d} \int_{\Omega}\left|\frac{\partial v_{i}}{\partial x_{j}}+\frac{\partial v_{j}}{\partial x_{i}}\right|^{2} \mathrm{~d} x \\
& =\frac{1}{4} \sum_{i, j=1}^{d} \int_{\Omega}\left|\frac{\partial v_{i}}{\partial x_{j}}\right|^{2}+2 \frac{\partial v_{i}}{\partial x_{j}} \frac{\partial v_{j}}{\partial x_{i}}+\left|\frac{\partial v_{j}}{\partial x_{i}}\right|^{2} \mathrm{~d} x \\
& =\frac{1}{2} \sum_{i, j=1}^{d} \int_{\Omega}\left|\frac{\partial v_{i}}{\partial x_{j}}\right|^{2}+\frac{\partial v_{i}}{\partial x_{j}} \frac{\partial v_{j}}{\partial x_{i}} \mathrm{~d} x \\
& =\frac{1}{2}\left(\|\nabla v\|_{L^{2}(\Omega)}^{2}+\|\operatorname{div} v\|_{L^{2}(\Omega)}^{2}\right) \\
& \geq \frac{1}{2}(2 \pi)^{d} \sum_{k \in \mathbb{Z}^{d} \backslash\{0\}}|k|^{2}|\hat{v}(k)|^{2} \\
& \geq \frac{1}{2}(2 \pi)^{d} \sum_{k \in \mathbb{Z}^{d} \backslash\{0\}}|\hat{v}(k)|^{2}=\frac{1}{2}\|v\|_{L^{2}(\Omega)}^{2} .
\end{aligned}
$$

Thus, by a density argument, we deduce the inequality

$$
\|v\|_{L^{2}(\Omega)} \leq \sqrt{2}\|D(v)\|_{L^{2}(\Omega)} \quad \forall v \in\left[W_{*}^{1,2}(\Omega)\right]^{d} .
$$

Since both $\|D(v)\|_{L^{2}(\Omega)}^{2} \geq \frac{1}{2}\|\nabla v\|_{L^{2}(\Omega)}^{2}$ and $\|D(v)\|_{L^{2}(\Omega)}^{2} \geq \frac{1}{2}\|v\|_{L^{2}(\Omega)}^{2}$, we have, by adding these two inequalities and then taking the square root, the desired inequality:

$$
\|v\|_{W^{1,2}(\Omega)} \leq 2\|D(v)\|_{L^{2}(\Omega)} \quad \forall v \in\left[W_{*}^{1,2}(\Omega)\right]^{d} .
$$

That completes the proof.

We are now ready to prove the inf-sup condition (5). Given any $v_{N} \in V_{N} \backslash\{0\}$, we consider the function $T_{N} \in \Sigma_{N} \backslash\{0\}$ defined by $T_{N}=D\left(w_{N}\right)$, where $w_{N} \in V_{N}$ is the unique solution of the problem

$$
\left(D\left(w_{N}\right), D\left(z_{N}\right)\right)=\left(v_{N}, z_{N}\right) \quad \forall z_{N} \in V_{N} .
$$

We note that, indeed, $T_{N} \neq 0$; for, if it were the case that $T_{N}=0$, then we would have $\left(v_{N}, z_{N}\right)=0$ for all $z_{N} \in V_{N}$, and hence $v_{N}=0$, which would contradict our assumption that $v_{N} \in V_{N} \backslash\{0\}$. The existence and uniqueness of $w_{N}$ is a direct consequence of the Lax-Milgram theorem and Korn's inequality, as stated in Lemma A.2. Hence,

$$
\begin{aligned}
b\left(v_{N}, T_{N}\right) & :=\left(-v_{N}, \operatorname{div} T_{N}\right)=\left(-v_{N}, \operatorname{div} D\left(w_{N}\right)\right)=\left(D\left(v_{N}\right), D\left(w_{N}\right)\right) \\
& =\left(D\left(w_{N}\right), D\left(v_{N}\right)\right)=\left\|v_{N}\right\|_{L^{2}(\Omega)}^{2} .
\end{aligned}
$$

As $\left(-\operatorname{div} T_{N}, z_{N}\right)=\left(-\operatorname{div} D\left(w_{N}\right), z_{N}\right)=\left(D\left(w_{N}\right), D\left(z_{N}\right)\right)=\left(v_{N}, z_{N}\right)$ for all $z_{N} \in V_{N}$, we have, with $z_{N}=-\operatorname{div} T_{N}$, that

$$
\left\|\operatorname{div} T_{N}\right\|_{L^{2}(\Omega)}^{2}=\left(-\operatorname{div} T_{N},-\operatorname{div} T_{N}\right)=\left(v_{N},-\operatorname{div} T_{N}\right)=b\left(v_{N}, T_{N}\right) .
$$


Further, by Lemma A.2,

$$
\begin{aligned}
\left\|T_{N}\right\|_{L^{2}(\Omega)}^{2} & =\left\|D\left(w_{N}\right)\right\|_{L^{2}(\Omega)}^{2}=\left(v_{N}, w_{N}\right) \leq\left\|v_{N}\right\|_{L^{2}(\Omega)}\left\|w_{N}\right\|_{L^{2}(\Omega)} \\
& \leq \sqrt{2}\left\|v_{N}\right\|_{L^{2}(\Omega)}\left\|D\left(w_{N}\right)\right\|_{L^{2}(\Omega)}=\sqrt{2}\left\|v_{N}\right\|_{L^{2}(\Omega)}\left\|T_{N}\right\|_{L^{2}(\Omega)}
\end{aligned}
$$

whereby

$$
\left\|T_{N}\right\|_{L^{2}(\Omega)}^{2} \leq 2\left\|v_{N}\right\|_{L^{2}(\Omega)}^{2}=2 b\left(v_{N}, T_{N}\right) .
$$

Now, summing this last inequality and the equality

$$
\left\|\operatorname{div} T_{N}\right\|_{L^{2}(\Omega)}^{2}=b\left(v_{N}, T_{N}\right)=\left\|v_{N}\right\|_{L^{2}(\Omega)}^{2}
$$

yields that

$$
\begin{aligned}
b\left(v_{N}, T_{N}\right) & \geq \frac{1}{3}\left\|T_{N}\right\|_{H(\mathrm{div} ; \Omega)}^{2} \\
& \geq \frac{1}{3}\left\|T_{N}\right\|_{H(\mathrm{div} ; \Omega)}\left\|\operatorname{div} T_{N}\right\|_{L^{2}(\Omega)} \\
& =\frac{1}{3}\left\|T_{N}\right\|_{H(\mathrm{div} ; \Omega)}\left[b\left(v_{N}, T_{N}\right)\right]^{\frac{1}{2}} \\
& =\frac{1}{3}\left\|T_{N}\right\|_{H(\mathrm{div} ; \Omega)}\left\|v_{N}\right\|_{L^{2}(\Omega)} .
\end{aligned}
$$

Thus we have shown that for each $v_{N} \in V_{N} \backslash\{0\}$ there exists a $T_{N} \in \Sigma_{N}$ such that

$$
b\left(v_{N}, T_{N}\right) \geq \frac{1}{3}\left\|T_{N}\right\|_{H(\mathrm{div} ; \Omega)}\left\|v_{N}\right\|_{L^{2}(\Omega)} .
$$

This implies that

$$
\sup _{T_{N} \in \Sigma_{N} \backslash\{0\}} \frac{b\left(v_{N}, T_{N}\right)}{\left\|T_{N}\right\|_{H(\mathrm{div} ; \Omega)}} \geq \frac{1}{3}\left\|v_{N}\right\|_{L^{2}(\Omega)} \quad \forall v_{N} \in V_{N},
$$

and hence,

$$
\inf _{v_{N} \in V_{N} \backslash\{0\}} \sup _{T_{N} \in \Sigma_{N} \backslash\{0\}} \frac{b\left(v_{N}, T_{N}\right)}{\left\|v_{N}\right\|_{L^{2}(\Omega)}\left\|T_{N}\right\|_{H(\operatorname{div} ; \Omega)}} \geq \frac{1}{3} .
$$

We thus deduce that the inf-sup condition (5) holds, with $c_{\text {inf-sup }} \geq 1 / 3$.

Mathematical Institute of Charles University, Sokolovská 83, 18675 Prague, Czech Republic E-mail address: mbul8060@karlin.mff.cuni.cz

Mathematical Institute of Charles University, Sokolovská 83, 18675 Prague, Czech Republic

E-mail address: malek@karlin.mff.cuni.cz

Mathematical Institute, University of Oxford, Woodstock Road, Oxford OX2 6GG, United Kingdom

E-mail address: Endre.Suli@maths.ox.ac.uk 